\title{
BENDING FLOWS FOR SUMS OF RANK ONE MATRICES
}

\author{
HERMANN FLASCHKA AND JOHN MILLSON
}

\section{Contents}

1. Introduction 2

2. The moduli space of polvgons in $\mathcal{H}_{m+1} \quad 6$

2.1. Coadioint orbits 6

2.2. The space of closed polvgons $\quad 7$

3. Nonemptiness of the moduli spaces 8

3.1. Necessitv of the Triangle Inequalities 8

3.2. Semistabilitv and Sufficiency of the Triangle Inequalities 10

$\begin{array}{ll}\text { 4. Smoothness of the Moduli Spaces } & 12\end{array}$

4.1. Decomposable Polvgons 12

4.2. The critical sidelengths of closed polvgons 16

5. Bending Hamiltonians 19

5.1. Bending Flows 20

5.2. Involutivity 22

6. A Complete Set of Bending Flows 23

6.1. The Weinstein-Aronszain Formula 23

6.2. Gel'fand-Tsetlin Patterns 24

6.3. Constructing a polvgon with given GTs pattern 26

7. Angle Variables and Four-Point Functions 28

7.1. Four-point functions and polvgons 28

7.2. Construction of angle variables 30

8. The dualitv between the bending svstems and the Gel'fand-Tsetlin svstems on Grassma

9. Pieri's formula and the dualitv at the quantum level 41

9.1. The Weinstein-Aronszain and Pieri formulas 42

9.2. Dualitv at the quantum level 42

10. Appendix: Bending and Hitchin Hamiltonians 44

10.1. Hitchin Hamiltonians 44

10.2. Bending Hamiltonians are not Hitchin Hamiltonians 45

$\begin{array}{ll}\text { References } & 47\end{array}$ 


\section{INTRODUCTION}

The aim of this paper is to generalize results of Kapovich and Millson [KM96] and Klyachko Kly92 on the moduli space $M_{\mathbf{r}}$ of (closed) polygons in $\mathbb{R}^{3}$ with prescribed sidelengths $\mathbf{r}=\left(r_{1}, \ldots, r_{n}\right)$, polygons related by a Euclidean motion being identified. They showed that $M_{\mathbf{r}}$ is a (possibly singular) symplectic manifold, and introduced a class of commuting Hamiltonian flows, the so-called bending flows. These flows bend the polygon about the diagonals emanating from one fixed vertex. The part of the polygon to one side of the diagonal does not move, while the other part rotates at constant speed. The lengths of the diagonals are action variables which generate the bending flows; the conjugate angle variables are the dihedral angles between the fixed and the moving parts.

We generalize this picture by replacing vectors in $\mathbb{R}^{3}$ by positive semidefinite rank-one Hermitean matrices. These have the form $e=$ $r w \otimes w^{*}$, where $r>0$ and $w$ is a unit vector in $\mathbb{C}^{m+1}$. Explicitly, $e: v \mapsto$ $r(v, w) w$ where $(, \quad)$ is the standard positive definite Hermitean form on $\mathbb{C}^{m+1}$. The edges of a polygon will be $e_{i}=r_{i} w_{i} \otimes w_{i}^{*}, i=1, \ldots, n$, $r_{i}>0$ fixed, and closed will mean closed up to a multiple of the identity,

$$
e_{1}+\cdots+e_{n}=\Lambda \mathbb{I}
$$

Equality of traces forces $\Lambda=\left(r_{1}+\cdots+r_{n}\right) /(m+1)$. Polygons are identified if they are related by simultaneous rotation of the sides by an element of $\mathrm{U}(\mathrm{m}+1)$. Since $w \otimes w^{*}$ is unchanged if $w$ is multiplied by $\exp (\sqrt{-1} \theta)$, we may think of an edge $r w \otimes w^{*}$ as a weighted point in the projective space $\mathbb{C P}^{m}$, and of a polygon as a weighted configuration of points in $\mathbb{C P}^{m}$.

The paper has three parts.

(1) A study of the moduli space $M_{\mathbf{r}}$. Criteria for nonemptiness and nonsingularity of $M_{\mathrm{r}}$ are found.

(2) A generalization of bending flows and their action-angle coordinates.

(3) A relation between the bending flows and representations of $\mathrm{U}(\mathrm{m}+1)$; this is reminiscent of geometric quantization.

This is the logical progression of the material, but (1) stands alone, and (2), about the bending flows, can be read independently of the rest. We give a brief outline of each part.

If follows from general results of Kly98 (however we sketch a direct proof in what follows) that $M_{\mathbf{r}}$ is nonempty if, and only if, the side 
lengths $\mathbf{r}$ satisfy the generalized triangle inequalities,

$$
m r_{i} \leq r_{1}+\cdots+\hat{r}_{i}+\cdots r_{n}, \quad 1 \leq i \leq n .
$$

This defines a convex cone $C(n, m+1)$ in $\mathbb{R}^{n}$. When $n=3$, the generalized triangle inequalities are the usual conditions for $r_{1}, r_{2}, r_{3}$ to be the sides of a Euclidean triangle, namely

$$
r_{1} \leq r_{2}+r_{3}, \quad r_{2} \leq r_{1}+r_{3}, \quad r_{3} \leq r_{1}+r_{2} .
$$

The proof of necessity is elementary, and is given in $\S 3.1$. Sufficiency is deeper. Only an outline of the argument is given in $\S \S 3.2$ and 3.3 ; for details the reader is referred to the literature. It follows from standard results, see for example MFK, Theorem 8.3, that $M_{\mathbf{r}}$ is canonically homeomorphic to a weighted (by $\mathbf{r}$ ) complex analytic quotient of the $n$-fold product $\Pi_{1}^{n} \mathbb{C P}^{m}$. For $m=1$, i. e. for spatial polygons, the connection between weighted analytic quotients of $\mathbb{C P}^{1}$ and $M_{\mathbf{r}}$ was found independently in KM96 and Kly98. The weighted quotient is nonempty if and only if there exists a weighted semistable configuration of points on $\mathbb{C P}^{m}$. If $\mathbf{r}$ satisfies the strong triangle inequalities, then any $n$-tuple of points on $\mathbb{C P}^{m}$ in general position (these always exist) is semistable for the weights $\mathbf{r}$.

In the Euclidean case, it was shown in KM96 that $M_{\mathbf{r}}$ is smooth if there is no polygon contained in a line. The corresponding statement in our case is that $M_{\mathbf{r}}$ is smooth if there is no decomposable polygon. A polygon is decomposable, roughly speaking, if there are closed subpolygons contained in orthogonal subspaces of $\mathbb{C}^{m+1}$. The side lengths $\mathbf{r}$ for which a decomposable polygon exists lie on certain hyperplane sections of the cone $C(n, m+1)$ called walls. A connected component of the complement of the walls is a chamber. We prove that $M_{\mathbf{r}}$ and $M_{\mathbf{r}^{\prime}}$ are diffeomorphic if $\mathbf{r}, \mathbf{r}^{\prime}$ lie in the same chamber. The topology of $M_{\mathbf{r}}$ will change as $\mathbf{r}$ crosses a wall(see for example [Hu] and Goldin]).

Turning now to the bending flows, we remark first that the action variables, or Hamiltonians generating the "bending", are a natural generalization of the Euclidean case. There, one has $\mathbb{R}^{3} \equiv \mathfrak{s u}(2)$; a diagonal $A$ of a polygon is identified with an element $\hat{A} \in \mathfrak{s u}(2)$, and the length $\|A\|$ is just the positive eigenvalue of $\hat{A}$. In our generalization, the bending Hamiltonians are also the eigenvalues, $\lambda_{i j}, j=1, \ldots, m+1$, of the diagonals $A_{i}=e_{1}+\cdots+e_{i+1}$, and they generate $2 \pi$-periodic flows. These flows again leave part of the polygon fixed, and conjugate the other part by $\exp \left(\sqrt{-1} t E_{i j}\right)$, where $E_{i j}$ is the spectral projection for $\lambda_{i j}$. This is a kind of bending with "internal degrees of freedom". Enough of the $\lambda_{i j}$ are functionally independent to give action coordinates (only on a dense open set, however). The eigenvalues 
of $A_{i+1}$ and $A_{i}$ interlace; this is a direct consequence of the WeinsteinAronszajn formula from perturbation theory. The interlacing property can be pictured by a triangular organization of the $\lambda_{i j}$, starting with the eigenvalue $r_{1}$ of $e_{1}=r_{1} w_{1} \otimes w_{1}^{*}$ and working up:

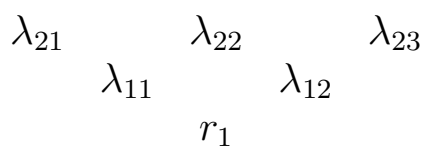

This is called a Gel'fand-Tsetlin pattern. As long as these inequalities are respected, the $\lambda_{i j}$ can be prescribed arbitrarily. Gel'fand-Tsetlin patterns were introduced in the Euclidean context by Hausmann and Knutson [HK97]. They showed, and this extends easily to our setting, that the bending flows and the Gel'fand-Tsetlin flows of Guillemin and Sternberg GS83] are dual to each other (via the Gel'fand-MacPherson duality GGMS]).

The angle variables also extend the Euclidean case (dihedral angles), but in a rather more subtle way. For vectors $w, x, y, z$ in $\mathbb{C}^{m+1}$, one defines the four-point function BeSch

$$
F(w, x, y, z)=(w, x)(x, y)(y, z)(z, w) .
$$

It is independent of the phases of its arguments, and so $\arg F$ is well defined on $\mathbb{C P}^{m}$. The arguments of $F$ in our setting will be the $w_{i}$ that define the edges $e_{i}=r w_{i} \otimes w_{i}^{*}$, and eigenvectors $u_{i j}$ corresponding to $\lambda_{i j}$. These are only defined up to phase, but using $\arg F\left(w_{i+1}, u_{i j}, w_{i+2}, u_{i, j+1}\right)$ we get global angle variables (on a dense open set again). In the Euclidean case, this amounts to a rather complicated way of expressing a dihedral angle. The proof of the Poisson bracket relations, \{angle , angle $\}=0$, etc., takes up all of $\S 7$.

The connection between bending flows and representation theory follows the ideas of Guillemin and Sternberg GS83. In Bohr-Sommerfeld quantization, one asks that the action variables take on integral values. If the $r_{i}, \lambda_{i j}$, and $\left(\sum_{i} r_{i}\right) /(m+1)$ are integers, the interlacing property of the $\lambda_{i j}$ reproduces the Pieri formula for the decomposition of tensor products of symmetric powers

$$
\bigotimes \mathcal{S}^{r_{i}}\left(\mathbb{C}^{m+1}\right)
$$

of the basic representation of $\mathrm{U}(\mathrm{m}+1)$ on $\mathbb{C}^{m+1}$.

The Hausmann-Knutson duality also has a representation-theoretic meaning. Gel'fand-Tsetlin patterns were invented to parameterize bases for vector spaces carrying representations of unitary (and general linear) groups. The possible patterns $(*)$ built from (integer) eigenvalues 
of successive diagonals index vectors of weight $\mathbf{r}=\left(r_{1}, \ldots, r_{n}\right)$ in the (Grassmannian) representation of $\mathrm{U}(\mathrm{n})$ with highest weight

$(* * *)$

$$
(\underbrace{\Lambda, \ldots, \Lambda}_{m+1}, 0, \ldots, 0) .
$$

We conclude that the multiplicity of the one-dimensional representation $\operatorname{det}^{\Lambda}$ in the tensor product $(* *)$ of $\mathrm{U}(\mathrm{m}+1)$ representations equals the multiplicity of the weight $\mathbf{r}$ in the representation $(* * *)$ of $U(n)$.

It must be said that these results are based on counting lattice points in convex polytopes and comparing their number with multiplicities known in representation theory. We do not construct actual representation spaces by any quantization method.

It is our hope that there are analogous results for all symplectic quotients of products of flag manifolds. In general for such products, one can find integrable systems that reduce to ours in the case of projective space, but it appears very hard to construct an explicit family of Hamiltonians with periodic flows, i.e. action variables. If such a construction could be carried out and the associated momentum polytope could be computed, then by counting lattice points in the momentum polytope one could find information on decomposing tensor products of irreducible representations. Many deep connections are now known between tensor product decompositions and convex polyhedra; these, however, do not seem to arise as images of momentum mappings. One of the main motivations for our paper is that the special case treated here is probably the only case where everything can be worked out with simple explicit formulas.

We conclude by noting that the spaces $M_{\mathbf{r}}$ studied in this paper were the subject of the book, [DO]. The study of the spaces $M_{\mathbf{r}}$ in DO was from the point of view of algebraic geometry and combinatorics, necessitating the restriction to the case in which the $r_{i}$ 's were integral (moreover the authors assumed that all the $r_{i}$ 's were equal). There appear to be interesting relations between our work and theirs.

Acknowledgements. We thank the referee for detailed and helpful comments, and especially for pointing out an error in the original version of this paper. In the appendix we answer a question posed by the referee concerning the relation between the duality of integrable systems of this paper and those of [AHH]. We would also like to thank Ron Donagi for some helpful conversations about Hitchin Hamiltonians. He suggested to us that if the bending Hamiltonians belonged to the Hitchin system then the underlying curve would have to degenerate (see the appendix). Finally, we thank Ben Howard for drawing 
our attention to confusion caused by a missing factor of 2 . The second author was supported in part by NSF grant DMS 01-04006.

\section{The moduli space of POlygons in $\mathcal{H}_{m+1}$}

In this section, we collect the notation used throughout, and in particular, introduce the moduli space of polygons with which we will be concerned.

\subsection{Coadjoint orbits.}

(1) Let $\mathcal{H}_{m+1}$ be the vector space of $(m+1) \times(m+1)$ Hermitean matrices. We identify it with the dual of the Lie algebra $\mathfrak{u}(m+1)$ via the pairing $\langle\xi, X\rangle=\operatorname{Im} \operatorname{Tr} \xi X$, for $\xi \in \mathfrak{u}(m+1), X \in \mathcal{H}_{m+1}$.

(2) $\mathcal{H}_{m+1}^{0}=\left\{X \in \mathcal{H}_{m+1} \mid \operatorname{Tr} X=0\right\}$. It is the dual of $\mathfrak{s u}(m+1)$.

(3) The gradient $\nabla f(X) \in \mathfrak{u}(m+1)$ of a smooth function $f$ on $\mathcal{H}_{m+1}$ is defined by

$\operatorname{Im} \operatorname{Tr}(\nabla f(X) Y)=\left.\frac{d}{d t}\right|_{t=0} f(X+t Y), \quad$ for all $Y \in \mathcal{H}_{m+1}$.

(4) A $\mathrm{U}(\mathrm{m}+1)$-orbit $\mathcal{O} \subset \mathcal{H}_{m+1}$ carries the Kostant-Kirillov symplectic form $\omega_{K K}$ defined by

$$
\omega_{K K}(X)([\xi, X],[\eta, X])=\operatorname{Im} \operatorname{Tr}(X[\xi, \eta]) .
$$

The Lie-Poisson bracket is

$$
\{f, g\}(X)=\operatorname{Im} \operatorname{Tr}(X[\nabla f(X), \nabla g(X)]),
$$

and Hamilton's equations have the form

$$
\dot{X}=[\nabla f(X), X] \text {. }
$$

In $\S 7.1$, we use the identification between $\mathcal{H}_{2}^{0}$ with bracket (2.1.1), and Euclidean space $\mathbb{R}^{3}$ with its standard Poisson bracket.

(5) Let $w \in \mathbb{C}^{m+1}$ be a unit vector. Define $w \otimes w^{*} \in \mathcal{H}_{m+1}$ by $w \otimes w^{*}(v)=(v, w) w$; it is a rank one projection. The matrices $r w \otimes w^{*}, r>0$, form an orbit $\mathcal{O}_{r}$ of $\mathrm{U}(\mathrm{m}+1)$. They will be "edges" of polygons, and are denoted by the letter $e$. Given $e \in \mathcal{O}_{r}$, the unit vector $w$ is determined up to multiplication by a complex number of modulus one. Hence $\mathcal{O}_{r}$ is diffeomorphic to $\mathbb{C P}^{m}$. As symplectic manifolds, they are related by $\omega_{K K}=$ $2 r \omega_{F S}$, where $\omega_{F S}$ is the Fubini-Study form on $\mathbb{C P}^{m}$.

Remark 2.1.1. For completeness, we verify the last assertion about the symplectic forms. The Fubini-Study metric is the $U(m+1)$-invariant Kähler metric normalized so that the holomorphic sectional curvature is 4 . To determine the scalar multiple relating two $U(m+1)$ invariant 
symplectic forms on $\mathbb{C P}^{m}$ it suffices to compute the period of each over a (complex linearly) embedded projective line $\mathbb{C P}^{1} \subset \mathbb{C P}^{m}$. For the Fubini-Study metric, a projective line has curvature 4, and is therefore isometric to a sphere of radius $1 / 2$ and area $\pi$. We may obtain a such a $\mathbb{C P}^{1}$ by embedding $\mathcal{H}_{2}^{0} \subset \mathcal{H}_{m+1}$ into the principal $2 \times 2$ block. Thanks to the identification of $\mathcal{H}_{2}^{0}$ with $\mathbb{R}^{3}$, it now suffices to relate the KostantKirillov form on an orbit of $\mathfrak{s u}(2)$ on $\mathbb{R}^{3}$, i. e. on a sphere $S_{r}^{2}$ of radius r, to the usual area form $d A$. The calculation in [MR, p. 412] shows that $\omega_{K K}\left(S_{r}^{2}\right)=d A / 2 r$. The symplectic area is $2 \pi r$, which agrees with the area given by the scaled Fubini-Study form, $2 r \omega_{F S}$, on the embedded $\mathbb{C P}^{1} \subset \mathcal{O}_{r}$

2.2. The space of closed polygons. Let $\mathbf{r}=\left(r_{1}, r_{2}, \ldots, r_{n}\right)$ be an $n$-tuple of positive numbers. We define a (closed) polygon with sidelengths $\mathbf{r}$ to be an $n$-tuple $\mathbf{e}=\left(e_{1}, e_{2}, \ldots, e_{n}\right)$ such that for all $i, 1 \leq$ $i \leq n$ we have

- a) $e_{i} \in \mathcal{O}_{r_{i}}$

- b) $\sum_{1}^{n} e_{i}=\Lambda \mathbb{I}$.

Note that $\Lambda=\frac{1}{m+1} \sum_{1}^{m+1} r_{i}$ follows from equality of traces in b). We call the matrices $e_{i}$ the edges of the polygon e and $r_{i}$ the length of the edge $e_{i}$. Condition (b) says that the polygon e is closed, modulo the center of $\mathcal{H}_{m+1}$.

(1) When $\mathbf{r}$ is given, $\Lambda$ always stands for $\frac{1}{m+1} \sum r_{i}$. Sometimes the notation $\Lambda_{\mathbf{r}}$ is used to emphasize the dependence of $\Lambda$ on $\mathbf{r}$.

(2) Given $\mathbf{r}$, define $\widetilde{N}_{\mathbf{r}}$ to be the product symplectic manifold $\Pi_{1}^{n} \mathcal{O}_{r_{i}}$. The diagonal action of $\mathrm{U}(\mathrm{m}+1)$ on $\widetilde{N}_{\mathbf{r}}$ is Hamiltonian with momentum map $\mu_{\mathbf{r}}$ given by

$$
\mu_{\mathbf{r}}(\mathbf{e})=\sum_{1}^{n} e_{i}
$$

We refer to elements of $\widetilde{N}_{\mathbf{r}}$ as linkages; they may or may not be closed.

(3) Given $\mathbf{r}$, let

$$
\widetilde{M}_{\mathbf{r}}=\mu_{\mathbf{r}}^{-1}(\Lambda \mathbb{I})=\left\{\mathbf{e} \in \widetilde{N}_{\mathbf{r}} \mid \sum_{i=1}^{n} e_{i}=\Lambda \mathbb{I}\right\} .
$$

The elements of $\widetilde{M}_{\mathbf{r}}$ are the polygons, or closed polygons for emphasis. The unitary group acts diagonally on $\widetilde{M}_{\mathbf{r}}$. We let i : $\widetilde{M}_{\mathbf{r}} \rightarrow \widetilde{N}_{\mathbf{r}}$ be the inclusion. 
(4) Finally, we define the moduli space, $M_{\mathbf{r}}$, of polygons (with sidelengths $\mathbf{r}$ ) to be the quotient of $\widetilde{M}_{\mathbf{r}}$ by the diagonal action of $\mathrm{U}(\mathrm{m}+1)$.

Because the stabilizer of the scalar matrix $\Lambda \mathbb{I}$ is all of $\mathrm{U}(\mathrm{m}+1)$, we obtain

Lemma 2.2.1. $M_{\mathbf{r}}$ is the symplectic quotient of $\widetilde{N}_{\mathbf{r}}$ corresponding to the (one-point) orbit $\Lambda \mathbb{I} \in \mathcal{H}_{m+1}$ under the diagonal action of $U(m+1)$.

\section{Nonemptiness of THE MOduli SPACES}

A simple set of inequalities on the side-lengths $r_{i}$ is necessary and sufficient for the moduli space to be nonempty. The elementary proof of necessity is given first. Sufficiency is deeper, and is based on the interpretation of polygons as weighted sets of points in projective space. For sake of completeness, that argument will be summarized in the Subsection 3.2, with references to literature where details may be found.

\subsection{Necessity of the Triangle Inequalities.}

Theorem 3.1.1. The moduli space $M_{\mathbf{r}}$ is nonempty if and only if $\mathbf{r}$ satisfies the system of inequalities

$$
m r_{i} \leq r_{1}+r_{2}+\cdots+\widehat{r}_{i}+\cdots+r_{n}, 1 \leq i \leq n .
$$

Here $\widehat{r}_{i}$ means that $r_{i}$ has been omitted in the summation.

Remark 3.1.2. We will call this system of inequalities (together with the inequalities $r_{i} \geq 0,1 \leq i \leq n$ ) the strong triangle inequalities of weight $m$. When $m=1$, they give the familiar conditions $r_{1} \leq r_{2}+r_{3}$, $r_{2} \leq r_{1}+r_{3}, r_{3} \leq r_{1}+r_{2}$ on the side lengths of a planar triangle. We will omit reference to the weight $m$ when it is clear from the context. Note that if we define $\rho=\sum_{i} r_{i}$ then the $i$-th inequality above is equivalent to

$$
r_{i} \leq \frac{1}{m+1} \rho
$$

We now turn to the proof of the necessity of the triangle inequalities.

Definition 3.1.3. Let $X \in \mathcal{H}_{m+1}^{0}$. Say that $X$ is maximally singular if $X$ is conjugate to a diagonal matrix with eigenvalues $\left(r,-\frac{r}{m}, \ldots,-\frac{r}{m}\right)$. We note that the orbit $\mathcal{O}_{r}^{0}$ under $U(m+1)$ of such an $X$ is the projection onto tracefree matrices of the orbit $\mathcal{O}_{r}$ through $\operatorname{diag}(r, 0, \ldots, 0)$.

Lemma 3.1.4. Suppose $X_{1}, X_{2} \in \mathcal{H}_{m+1}^{0}$ are distinct, maximally singular, and satisfy $\operatorname{Tr}\left(X_{j}^{2}\right)=1$. Then $\operatorname{Tr}\left(X_{1} X_{2}\right) \geq-1 / m$, with equality if and only if $X_{1}$ and $X_{2}$ commute. 
Proof. We may write

$$
X_{j}=\sqrt{\frac{m+1}{m}}\left(w_{j} \otimes w_{j}^{*}-\frac{1}{m+1} \mathbb{I}\right),
$$

where $\left\|w_{j}\right\|=1, j=1,2$.

Then

$$
\begin{aligned}
\operatorname{Tr} X_{1} X_{2} & =\frac{m+1}{m} \operatorname{Tr}\left[\left(w_{1} \otimes w_{1}^{*}-\frac{1}{m+1} \mathbb{I}\right)\left(w_{2} \otimes w_{2}^{*}\right)\right] \\
& =\frac{m+1}{m}\left[\left|\left(w_{1}, w_{2}\right)\right|^{2}-\frac{1}{m+1}\right] \geq \frac{m+1}{m} \cdot-\frac{1}{m+1} \\
& =-\frac{1}{m} .
\end{aligned}
$$

Clearly we have equality if and only if $\left(w_{1}, w_{2}\right)=0$ if and only if $X_{1}$ and $X_{2}$ commute.

Proposition 3.1.5. Suppose that $M_{\mathbf{r}}$ is nonempty. Then $\mathbf{r}$ satisfies the strong triangle inequalities of weight $m$.

Proof. Choose e $\in \widetilde{M}_{\mathbf{r}}$. Then $e_{1}+\cdots+e_{n}=\Lambda \mathbb{I}$ is equivalent to $r_{1} X_{1}+\cdots+r_{n} X_{n}=0$, where the matrices

$$
X_{j}=\sqrt{\frac{m+1}{m}}\left(w_{j} \otimes w_{j}^{*}-\frac{1}{m+1} \mathbb{I}\right)
$$

satisfy the hypotheses of Lemma 3.1.4. Alternatively,

$$
r_{i} X_{i}=-r_{1} X_{1}-\cdots-\widehat{r}_{i} X_{i}-\cdots-r_{n} X_{n} .
$$

Multiply each side by $X_{i}$ and take the trace to obtain

$$
r_{i}^{2}=-\sum_{j(\neq i)} r_{i} r_{j} \operatorname{Tr}\left(X_{j} X_{i}\right) \leq \frac{1}{m} \sum_{j(\neq i)} r_{i} r_{j} .
$$

Now divide both sides by $r_{i}$ to obtain the result.

The generalized triangle inequalities define a cone in $\left(\mathbb{R}_{+}\right)^{n}$ :

\section{Definition 3.1.6.}

$$
C(n, m+1)=\left\{\mathbf{r} \in\left(\mathbb{R}_{+}\right)^{n} \mid M_{\mathbf{r}} \neq \emptyset\right\} .
$$

The intersection of $C(n, m+1)$ with the hyperplane $\sum r_{i}=m+1$ is known in the literature as the hypersimplex. Side lengths $\mathbf{r}$ for which the moduli space $M_{\mathbf{r}}$ is singular will be shown to lie on certain hyperplane sections of $C(n, m+1)$.

We next give the outline of the proof that $M_{\mathrm{r}}$ is nonempty when $\mathbf{r} \in C(n, m+1)$. The method is based on the identification of an edge $r_{i} w_{i} \otimes w_{i}^{*}$ with a point $\mathbb{C} w_{i} \in \mathbb{C P}^{m}$ of weight $r_{i}$. The nonemptiness of 
the moduli space of such weighted points follows from a comprehensive general theory.

\subsection{Semistability and Sufficiency of the Triangle Inequalities.}

3.2.1. Analytic quotients and symplectic quotients. In [Sj95 and HL94, the authors constructed the analytic quotient of a (not necessarily projective) compact Kähler manifold $M$ by the action of a complex reductive group $G$. It is assumed that some maximal compact subgroup $K \subset G$ acts in a Hamiltonian fashion on $M$ with momentum map $\mu$. In their theory, a point $m \in M$ is defined to be semistable if the closure of the orbit $G \cdot m$ intersects the subset $\mu^{-1}(0)$ of $M$. The set of semistable points is denoted by $M^{s s t}$; it is open in $M$. A point of $M$ is defined to be nice semistable if the orbit itself intersects $\mu^{-1}(0)$. Define an equivalence relation, called extended orbit equivalence, by declaring two points to be related if their orbit closures intersect. (That this is indeed an equivalence relation follows from a theorem asserting that each equivalence class of semistable points contains a unique nice semistable orbit). We emphasize that the notion of semistability depends on the symplectic structure, i.e. on $\mathbf{r}$ in our case.

The analytic quotient of $M$ by $G$, denoted $M / / G$, is then defined to be the quotient of $M^{\text {sst }}$ by extended orbit equivalence. It is a hard theorem of Sj95 and HL94 that the resulting quotient topological space is Hausdorff and compact, and in fact has a canonical structure of a complex analytic space.

Since, by definition, any point in $\mu^{-1}(0)$ is (nice) semistable, there is an induced map from the symplectic quotient $\mu^{-1}(0) / K$ to the analytic quotient. Sj95 and HL94 prove that this map is a homeomorphism.

These results were proved earlier for smooth quotients and when the quotient has only orbifold singularities (i.e. the stabilizer of every $x \in \mu^{-1}(0)$ is finite) in [Ki], Theorem 7.5, and also for general point stabilizers if $M$ is a smooth complex projective variety and the symplectic form represents the (dual of the) hyperplane section class in Ki], Remark 8.14. In our setting, this amounts to assuming that either $\mathbf{r}$ is not on a wall or that $\mathbf{r}$ is integral (it is probable that some weakening of this condition will still result in an integral Kähler class). In this case the analytic quotient $M / / G$ is a complex projective variety.

We have seen that our space $M_{\mathbf{r}}$ is a symplectic quotient of $\left(\mathbb{C P}^{m}\right)^{n}$ by $\mathrm{U}(\mathrm{m}+1)$, where the $i^{\text {th }}$ factor is given the symplectic structure which is $2 r_{i}$ times the usual Fubini-Study form. In the next subsection we will describe the corresponding analytic quotient of $\left(\left(\mathbb{C P}^{m}\right)^{n}\right.$ by $\mathrm{GL}(\mathrm{m}+1, \mathbb{C})$ in the sense indicated above. In particular, when $\mathbf{r}$ is 
integral then $M_{\mathbf{r}}$ will have a canonical structure of a complex projective variety.

3.2.2. Weighted semistable configurations on $\mathbb{C P}^{m}$. In this subsection we describe the semistable configurations on $\left(\mathbb{C P}^{m}\right)^{n}$ equipped with the $\mathbf{r}-$ dependent symplectic structure just described. Set $[n]=\{1,2, \ldots, n\}$.

Definition 3.2.1. A configuration of $n$ points on $\mathbb{C P}^{m}$ is a map $f$ from $[n]$ to $\mathbb{C P}^{m}$.

Let $\nu_{\mathbf{r}}$ be the measure on $[n]$ that assigns mass $r_{i}$ to the point $i$. Also, recall that we have defined $\rho=\sum r_{i}$.

The proof of the following theorem is left to the reader. For the case of integral weights it is one of the standard results in Geometric Invariant Theory. For example, when all weights are $\frac{1}{n+1}($ so $\rho=1)$, it is proved in [MFK, Definition 3.7/Proposition 3.4].

Theorem 3.2.2. A configuration $f$ on $\mathbb{C P}^{m}$ is semistable if and only if

$$
f_{*} \nu_{\mathbf{r}}(L) \leq \frac{\operatorname{dim} L+1}{m+1} \rho
$$

for any linear subspace $L$ of $\mathbb{C P}^{m}$

Remark 3.2.3. In this inequality, the left side is the mass of the closed subset $L \subset \mathbb{C P}^{m}$ for the push-forward measure $f_{*} \nu_{\mathbf{r}}$. Intuitively, these semistability inequalities say that not too many points can coincide, not too many can lie on a line, not too many on a plane etc.

The configuration $f$ is said to be "in general position" if no two points coincide, at most two points lie on a line, at most three on a plane, ..., at most $k+1$ lie in a projective subspace of dimension $k$. The set of configurations in general position is a nonempty Zariskidense open subset of $\left(\mathbb{C P}^{m}\right)^{n}$. In particular, such configurations exist. Thus the result that $\mathbf{r}$ satisfies strong triangle inequalities implies $M_{\mathbf{r}}$ is nonempty is an immediate consequence of the following

Proposition 3.2.4. Suppose that $f$ is in general position. Then $f$ is weighted semistable if and only if $\mathbf{r}$ satisfies the generalized triangle inequalities.

Proof. Since any subset of $k$ points in general position spans a projective subspace of projective dimension $k-1$ it is an immediate consequence of Theorem 3.2 .2 that $f$ is semistable if and only if for all $I \subset\{1,2, \cdots, n\}$

$$
\sum_{i \in I} r_{i} \leq \frac{|I|}{m+1} \rho .
$$


Clearly, the resulting system of inequalities contains, and is implied by, the subset in which $|I|=1$ :

$$
r_{i} \leq \frac{1}{m+1} \rho
$$

We have already noted (equation (3.1.1)) that this system is equivalent to the system of strong triangle inequalities.

Since configurations in general position always exist, we have the missing implication in Theorem 3.1.1

Corollary 3.2.5. $M_{\mathbf{r}}$ is nonempty if $\mathbf{r}$ satisfies the strong triangle inequalities.

\section{Smoothness of the Moduli Spaces}

In this section we give a sufficient condition in terms of $\mathbf{r}$ for the space $M_{\mathbf{r}}$ to be smooth.

4.1. Decomposable Polygons. For $m=1$, it was shown in KM96] that $M_{\mathbf{r}}$ will have singularities if, and only if, the index set $\{1, \ldots, n\}$ can be partitioned into proper subsets $I, J$ so that

$$
\sum_{i \in I} r_{i}=\sum_{j \in J} r_{j}
$$

Then there exists a polygon (in Euclidean space), with the given side lengths $\mathbf{r}$, that is contained in a line segment; such a polygon was called degenerate. It was further proved in KM96] that a polygon is a singular point of $M_{\mathbf{r}}$ if and only if it is degenerate. We need analogs of (4.1.1) and of the notion of degenerate polygon for the case $m \geq 2$.

Definition 4.1.1. For $1 \leq k \leq m$ and $I \cup J$ a proper partition of $\{1, \ldots, n\}$, define the hyperplane

$$
H_{I, J, k}=\left\{\mathbf{r} \in \mathbb{R}_{+}^{n} \mid k \sum_{i \in I} r_{i}=(m-k+1) \sum_{j \in J} r_{j}\right\} .
$$

(Note that this reduces to 4.1.1) when $m=1$ ). The wall corresponding to this hyperplane is the intersection

$$
W_{I, J, k}=H_{I, J, k} \cap C(n, m+1)
$$

(cf. Definition 3.1.6).

Notation 4.1.2. We will write $I=\left\{i_{1}, \ldots, i_{p}\right\}, J=\left\{j_{1}, \ldots, j_{k}\right\}, p+q=$ $n$, and take $I$ and $J$ to be ordered, $i_{1}<i_{2}<\ldots, j_{1}<j_{2}<\ldots$. Set $\mathbf{r}_{I}=\left(r_{i_{1}}, \ldots, r_{i_{p}}\right)$, and likewise for $J$. Let $\rho_{I}=\sum_{i \in I} r_{i}$ (similarly for 
$\left.\rho_{J}\right)$, and define $\Lambda_{I}=\rho_{I} /(m-k+1), \Lambda_{J}=\rho_{J} / k$, by analogy with $\Lambda=\rho /(m+1)$.

Lemma 4.1.3. Suppose that $\mathbf{r} \in W_{I, J, k}$. Then $\mathbf{r}_{I}$ (resp. $\mathbf{r}_{J}$ ) satisfies the strong triangle inequalities with weight $m-k$ (resp $k-1)$. Explicitly:

$$
\begin{aligned}
& r_{i} \leq \frac{1}{m-k+1} \rho_{I}, \text { for all } i \in I \\
& r_{j} \leq \frac{1}{k} \rho_{J}, \text { for all } j \in J .
\end{aligned}
$$

Proof. We show that $\mathbf{r}_{I}$ satisfies the strong triangle inequalities with weight $m-k$. According to Definition 4.1.1. $k \rho_{I}=(m-k+1) \rho_{J}$. Obviously, $\rho_{I}+\rho_{J}=\sum r_{i}=\rho$. Solving these two equations for $\rho_{I}$, we get $(m+1) \rho_{I}=(m-k+1) \rho$, or

$$
\frac{\rho_{I}}{m-k+1}=\frac{\rho}{m+1}
$$

Since $\mathbf{r}$ already satisfies the strong triangle inequalities of weight $m$, equation (3.1.1) shows the right side to be greater than $r_{i}$. This gives the desired inequality for $\rho_{I}$; the proof for $\rho_{J}$ is similar.

Lemma 4.1.4. If $\mathbf{r} \in W_{I, J, k}$, then $\Lambda_{I}=\Lambda_{J}=\Lambda$.

Proof. Since $\mathbf{r} \in W_{I, J, k}$, we have $k \rho_{I}=(m-k+1) \rho_{J}$, which implies $\Lambda_{I}=\Lambda_{J}$. Furthermore,

$$
k \rho=k \rho_{I}+k \rho_{J}=(m-k+1) \rho_{J}+k \rho_{J}=(m+1) \rho_{J},
$$

whence $\Lambda=\rho /(m+1)=\rho_{J} / k=\Lambda_{J}$.

We will see that if $\mathbf{r}$ does not lie on a wall, then $M_{\mathbf{r}}$ is smooth. To this end, we need the analog of degenerate polygon. It is the "decomposable polygon", in which the edges indexed by $I$ and $J$ act in orthogonal subspaces.

Let $\mathbf{r} \in W_{I, J, k}$. Choose an orthogonal decomposition $\mathbb{C}^{m+1}=V_{1} \oplus V_{2}$ with $\operatorname{dim} V_{1}=m-k+1$ and $\operatorname{dim} V_{2}=k$. Let $\mathcal{H}_{i}$ denote the set of Hermitean endomorphisms of $V_{i}$. We have inclusions $\alpha_{i}: \mathcal{H}_{i} \rightarrow \mathcal{H}_{m+1}$ given by

$$
\alpha_{i}(X)=\iota_{V_{i}} \circ X \circ \pi_{V_{i}}
$$

where $\iota_{V_{i}}$ is the inclusion of $V_{i}$ into $V$ and $\pi_{V_{i}}$ is the orthogonal projection from $V$ to $V_{i}$. Note that if $w \in V_{i}$, then $w \otimes w^{*}$ is in the image of $\alpha_{i}$.

We wish to define a map

$$
\iota_{I, J, V_{1}, V_{2}}: \widetilde{M}_{\mathbf{r}_{I}}\left(\mathcal{H}_{1}\right) \times \widetilde{M}_{\mathbf{r}_{J}}\left(\mathcal{H}_{2}\right) \rightarrow \widetilde{N}_{\mathbf{r}} .
$$

Let $\sigma$ be the $p, q$ shuffle permutation given by 


$$
\begin{aligned}
\sigma(k) & =i_{k}, \quad 1 \leq k \leq p \\
\sigma(p+l) & =j_{l}, \quad 1 \leq l \leq q .
\end{aligned}
$$

Choose polygons

$$
\mathbf{e}^{(1)}=\left(e_{1}^{(1)}, \cdots, e_{p}^{(1)}\right) \in \widetilde{M}_{\mathbf{r}_{I}}\left(\mathcal{H}_{1}\right), \mathbf{e}^{(2)}=\left(e_{1}^{(2)}, \cdots, e_{q}^{(2)}\right) \in \widetilde{M}_{\mathbf{r}_{I}}\left(\mathcal{H}_{2}\right) .
$$

Such polygons exist, since $\mathbf{r}_{I}$ and $\mathbf{r}_{J}$ satisfy the strong triangle inequalities (Lemma 4.1.3).

Then we define $\overline{\mathbf{e}}:=\iota_{I, J, V_{1}, V_{2}}\left(\mathbf{e}^{(1)}, \mathbf{e}^{(2)}\right)$ by

$$
\begin{aligned}
& \bar{e}_{i_{k}}=\alpha_{1}\left(e_{k}^{(1)}\right), 1 \leq k \leq p \\
& \bar{e}_{j_{l}}=\alpha_{2}\left(e_{l}^{(2)}\right), \quad 1 \leq l \leq q .
\end{aligned}
$$

We then have $\overline{\mathbf{e}}=\overline{\mathbf{e}}_{I} \oplus \overline{\mathbf{e}}_{J}$, and it follows from Lemma 4.1.4 that $\overline{\mathbf{e}} \in \widetilde{M}_{\mathbf{r}}$. Therefore the image of $\iota_{I, J, V_{1}, V_{2}}$ lies in $\widetilde{M}_{\mathbf{r}}$, i. e. , consists of closed polygons.

Definition 4.1.5. We say that $\mathbf{e} \in \widetilde{M}_{\mathbf{r}}$ is decomposable if it lies in the image of the map $\iota_{I, J, V_{1}, V_{2}}$ for some choice of $I, J, V_{1}, V_{2}$ as above.

Proposition 4.1.6. $\widetilde{M}_{\mathbf{r}}$ contains a decomposable polygon if, and only if, $\mathbf{r}$ lies on a wall.

Proof. Suppose that e is decomposable. We use the notation above. Since $\mathbf{e}_{I}$ is closed, $\sum_{I} e_{i}=\Lambda_{I} \mathbb{I}_{1}$, where $\mathbb{I}_{1}$ is the identity in $\operatorname{End}\left(V_{1}\right)$. Similarly $\sum_{J} e_{j}=\Lambda_{J} \mathbb{I}_{2}$. Because $\overline{\mathbf{e}}$ is closed, we have $\sum_{k=1}^{n} \bar{e}_{k}=\Lambda \mathbb{I}$ (II is the identity on $\mathbb{C}^{m+1}$ ). Clearly this last sum is also of block form $\Lambda_{I} \mathbb{I}_{1} \oplus \Lambda_{J} \mathbb{I}_{2}$ in $\operatorname{End}\left(\mathbb{C}^{m+1}\right)=\operatorname{End}\left(V_{1} \oplus V_{2}\right)$. Hence $\Lambda_{I}=\Lambda_{J}=\Lambda$. This implies $\mathbf{r} \in W_{I, J, k}$.

Conversely, suppose that $\mathbf{r} \in W_{I, J, k}$. By Lemma 4.1.3 and Theorem 3.1.1 there exist a closed $p$-gon $\mathbf{e}^{(1)} \in \mathcal{H}_{1}$ with side-lengths $\mathbf{r}_{I}$ and a closed $q$-gon $\mathbf{e}^{(2)} \in \mathcal{H}_{2}$ with side lengths $\mathbf{r}_{J}$. Then $\iota_{I, J, V_{1}, V_{2}}\left(\mathbf{e}^{(1)}, \mathbf{e}^{(2)}\right)$ is a decomposable polygon with side-lengths $\mathbf{r}$.

The next few lemmas are well known, but we will state and prove them because some of them will play a critical role later. As above, $\mathcal{H}_{j}$ denotes the Hermitean endomorphisms of a subspace $V_{j} \subset \mathbb{C}^{m+1}$ and $\alpha_{j}: \mathcal{H}_{j} \rightarrow \mathcal{H}_{m+1}$ is the natural inclusion.

For $\mathbf{e} \in \widetilde{N}_{\mathbf{r}}$, let $Z\left(e_{i}\right)$ denote the centralizer of $e_{i}$ in $\mathcal{H}_{m+1}^{0}$, and let $Z(\mathbf{e})=\bigcap_{i} Z\left(e_{i}\right)$.

Lemma 4.1.7. Suppose that $X \in Z\left(e_{i}\right)$, and let $\mathbb{C}^{m+1}=\oplus_{j=1}^{l} V_{j}$ be the eigenspace decomposition of $X$. Then there exists $j$ such that $e_{i} \in$ $\alpha_{j}\left(\mathcal{H}_{j}\right)$. 
Proof. If $e_{i}=r_{i} w_{i} \otimes w_{i}^{*}$, we have $\mathbb{C} w_{i}=\operatorname{ker}\left(e_{i}-r_{i} \mathbb{I}\right)$. Since $X$ and $e_{i}$ commute, $w_{i}$ is also an eigenvector of $X$. Hence $w_{i} \in V_{j}$ for some $j$ and $r_{i} w_{i} \otimes w_{i}^{*} \in \alpha_{j}\left(\mathcal{H}\left(V_{j}\right)\right)$.

Lemma 4.1.8. Let $\mathbf{e} \in \widetilde{N}_{\mathbf{r}}$. Then $\operatorname{Im} T_{\mathbf{e}} \mu_{\mathbf{r}} \subset \mathcal{H}_{m+1}^{0}$.

Proof. If $w(t)$ is a smooth curve in $\mathbb{C}^{m+1}$, with $\|w(t)\| \equiv 1$, then $\operatorname{Tr} w(t) \otimes w(t)^{*} \equiv 1$ implies $\operatorname{Tr} \frac{d}{d t}\left(w(t) \otimes w(t)^{*}\right) \equiv 0$. Hence the derivative of the momentum map $\mu_{\mathbf{r}}: \mathbf{e} \mapsto \sum_{i} r_{i} w_{i} \otimes w_{i}^{*}$ maps into $\mathcal{H}_{m+1}^{0}$.

Lemma 4.1.9. Let ${ }^{\perp}$ denote orthogonal complement in $\mathcal{H}_{m+1}^{0}$. Then, again for $\mathbf{e} \in \widetilde{N}_{\mathbf{r}}$

$$
\left(\operatorname{Im} T_{\mathbf{e}} \mu\right)^{\perp}=Z(\mathbf{e}) .
$$

Proof. Indeed, the derivative $T_{e} \mu_{\mathbf{r}}$ will be onto if, and only if,

$$
T_{e_{1}}\left(\mathcal{O}_{r_{1}}\right)+\cdots+T_{e_{n}}\left(\mathcal{O}_{r_{n}}\right)=\mathcal{H}_{m+1}^{0} .
$$

Thus $T_{e} \mu_{\mathbf{r}}$ is onto if, and only if,

$$
T_{e_{1}}\left(\mathcal{O}_{r_{1}}\right)^{\perp} \cap \ldots \cap T_{e_{n}}\left(\mathcal{O}_{r_{n}}\right)^{\perp}=\{0\} .
$$

But $T_{e_{i}}\left(\mathcal{O}_{r_{i}}\right)^{\perp}=\left\{\left[e_{i}, X\right] \mid X \in \mathcal{H}_{m+1}^{0}\right\}^{\perp}=Z\left(e_{i}\right)$, and the lemma follows.

Corollary 4.1.10. Let $\mathbf{e} \in \widetilde{N}_{\mathbf{r}}$. Then

$$
T_{\mathbf{e}} \mu_{\mathbf{r}}: T_{\mathbf{e}}\left(\tilde{N}_{\mathbf{r}}\right) \rightarrow \mathcal{H}_{m+1}^{0} \text { is not onto } \Longleftrightarrow Z(\mathbf{e}) \neq\{0\} .
$$

The next lemma relates surjectivity of the moment map to indecomposability.

Lemma 4.1.11. Now suppose that $\mathbf{e} \in \widetilde{M}_{\mathbf{r}}$. Then

$$
T_{\mathbf{e}} \mu_{\mathbf{r}} \text { is onto } \Longleftrightarrow \mathbf{e} \text { is not decomposable. }
$$

Proof. Suppose that $T_{\mathbf{e}} \mu_{\mathbf{r}}$ is not onto. Choose a nonzero $X \in Z(\mathbf{e})$. Suppose that $X$ has $\ell$ distinct eigenvalues, so that $\mathbb{C}^{m+1}$ is the orthogonal sum of the corresponding eigenspaces $W_{j}$. For each $e_{i}=r_{i} w_{i} \otimes w_{i}^{*}$, $w_{i} \in W_{j_{i}}$ for some $j_{i}$, by Lemma 4.1.7. Now set $V_{1}=W_{1}+\cdots+W_{\ell-1}$, $V_{2}=W_{\ell}$. Define $I=\left\{i \mid w_{i} \in V_{1}\right\}, J=\left\{j \mid w_{j} \in V_{2}\right\}$. It follows that $\mathbf{e}$ lies in the image of the map $\iota_{I, J, V_{1}, V_{2}}$ and so is decomposable.

Now suppose that $\mathbf{e}$ is decomposable. Then there exists an orthogonal splitting $V=V_{1} \oplus V_{2}$ and a partition $\{1, \cdots, n\}=I \cup J$ such that $\mathbf{e}=\mathbf{e}_{I}+\mathbf{e}_{J}$ is in the image of the map $\iota_{I, J, V_{1}, V_{2}}$. Let $X=\mathbb{I}_{1} \oplus \mathbf{0}$. Then $X \in Z(\mathbf{e})=\left(\operatorname{Im} T_{\mathbf{e}} \mu_{\mathbf{r}}\right)^{\perp}$.

Let $\widetilde{\Sigma}_{\mathbf{r}} \subset \widetilde{M}_{\mathbf{r}}$ denote the set of decomposable polygons. It is invariant under $\mathrm{U}(\mathrm{m}+1)$; let $\Sigma_{\mathbf{r}}$ be the image of $\widetilde{\Sigma}_{\mathbf{r}}$ in $M_{\mathbf{r}}$. 
Theorem 4.1.12. (i) $\widetilde{M}_{\mathbf{r}}-\widetilde{\Sigma}_{\mathbf{r}}$ is a smooth manifold. (ii) The group $S U(m+1)$ acts freely on $\widetilde{M}_{\mathbf{r}}-\widetilde{\Sigma}_{\mathbf{r}}$, hence the quotient $M_{\mathbf{r}}-\Sigma_{\mathbf{r}}$ is a smooth manifold.

Proof. Part (i) follows from one implication in Lemma 4.1.11, if e is not decomposable, then $\Lambda \mathbb{I}$ is a regular value of $\mu_{\mathbf{r}}$.

For (ii), we need to check that if $\mathbf{e}$ is not decomposable, then the stabilizer of e under the action of $\mathrm{U}(\mathrm{m}+1)$ is trivial. The argument in Lemma 4.1.11 still works, because we deal with matrix groups. If $\kappa \mathbf{e} \kappa^{-1}=\mathbf{e}$, we write $\mathbb{C}^{m+1}$ as sum of eigenspaces of $\kappa$, and proceed as before.

Corollary 4.1.13. If $\mathbf{r}$ does not lie on a wall, then $M_{\mathbf{r}}$ is a smooth manifold of dimension $2 m(n-m-2)$.

Proof. The smoothness of $M_{\mathbf{r}}$ follows from the theorem. To compute the dimension of $M_{\mathbf{r}}$, we note that $M_{\mathbf{r}}$ is the symplectic quotient of $\left(\mathbb{C P}^{m}\right)^{n}$ by the projective unitary group $P U(m+1)$. Thus we obtain

$$
\operatorname{dim}_{\mathbb{R}} M_{\mathbf{r}}=2 m n-2\left[(m+1)^{2}-1\right]=2 m n-2 m^{2}-4 m .
$$

4.2. The critical sidelengths of closed polygons. In this subsection, we study the map $\overline{\mathbf{s}}$ that maps a closed polygon e to the vector $\mathbf{r}$ of its side lengths, and show in Theorem 4.2.7 that its critical values are the union of the walls (Definition 4.1.1).

Notation 4.2.1. The space of not necessarily closed linkages in $\mathcal{H}_{m+1}$ with rank 1 positive semi-definite edges and arbitrary (positive) side lengths is denoted by

$$
\text { Pol }=\left\{\mathbf{e} \mid \mathbf{e} \in \tilde{N}_{\mathbf{r}} \text { for some } \mathbf{r}\right\} .
$$

We will use $\mu$ to denote the restriction of the momentum map $\mu$ for the diagonal action of $U(m+1)$ on $\mathcal{H}_{m+1}^{n}$ to Pol. (Recall that $\mu_{\mathbf{r}}$ is the moment map on linkages, closed or not, with given side lengths r). Further, introduce the subset of closed polygons with arbitrary side lengths,

$$
\mathrm{CPol}=\left\{\mathbf{e} \mid \mathbf{e} \in \widetilde{M}_{\mathbf{r}} \text { for some } \mathbf{r}\right\} \subset \mathrm{Pol} .
$$

The idea of the argument is this. If $\mathbf{e}(t)=\left(r_{1}(t) w_{1}(t) \otimes w_{1}(t)^{*}, \ldots\right)$ is a curve in CPol through $\mathbf{e}(0)=\mathbf{e}, \mathbf{r}(0)=\mathbf{r}$, the derivative of the moment map $\mu$ has the form

$$
T_{\mathbf{e}} \mu=\sum \dot{r}_{i}(0) e_{i}+\sum r_{i}\left(w_{i} \otimes \dot{w}_{i}(0)^{*}+\dot{w}_{i}(0) \otimes w_{i}^{*}\right) .
$$


The first sum is a linear combination of edges, while the second sum is in the image of the moment map $\mu_{\mathbf{r}}$. Since $T_{\mathbf{e}} \overline{\mathbf{s}}=\dot{\mathbf{r}}(0)$, the span of the edges relates surjectivity of $T_{\mathbf{e}} \mu$ with surjectivity of $T_{\mathbf{e}} \overline{\mathbf{s}}$.

Let $\mathbf{E} \subset \mathcal{H}_{m+1}$ be the span of the edges $e_{i}$ of the linkage $\mathbf{e}$.

Lemma 4.2.2. Suppose that $\mathbf{e} \in C$ Pol. Then

$$
Z(\mathbf{e}) \subset \mathbf{E} \text {. }
$$

Proof. Let $X \in Z(\mathbf{e})$, with eigenvalues $\lambda_{i}, i=1, \ldots, l$, and let $\mathbb{C}^{m+1}=$ $\oplus_{i=1}^{l} V_{i}$ be the eigenspace splitting of $\mathbb{C}^{m+1}$ under $X$. Then by Lemma 4.1.7. for each $i, 1 \leq i \leq n$, there exists $j_{i}$ such that $e_{i} \in \alpha_{j_{i}}\left(\mathcal{H}_{j_{i}}\right)$. Hence, e is decomposable with respect to this splitting. Thus there exists a permutation $\sigma$ such that $\mathbf{e}=\sigma\left(e_{1}^{(1)}, \cdots, e_{p_{1}}^{(1)}, \cdots, e_{1}^{(l)}, \cdots, e_{p_{l}}^{(l)}\right)$ with $e_{j}^{(i)}=r(i)_{j} w(i)_{j} \otimes\left(w_{j}^{(i)}\right)^{*}$, and $w_{j}^{(i)} \in V_{i}, 1 \leq i \leq l, 1 \leq j \leq p_{i}$.

Since $\mathbf{e}$ is closed, we have $\sum_{i} e_{i}=\Lambda$ II. As a consequence we have

$$
\sum_{j=1}^{p_{i}} e_{j}^{(i)}=\Lambda \Pi_{i}
$$

where $\Pi_{i}$ is the projection on $V_{i}$. Thus the $\Pi_{i}$ lie in the span $\mathbf{E}$. But since the splitting of $\mathbb{C}^{m+1}$ is the eigenspace decomposition of $X$, we have $X=\sum_{i=1}^{l} \lambda_{i} \Pi_{i}$. Thus $X \in \mathbf{E}$.

We do not need the next corollary in what follows but have included it for completeness. We let $\mathfrak{t}$ denote the abelian Lie subalgebra consisting of the diagonal matrices in $\mathcal{H}_{m+1}$.

Corollary 4.2.3. There exists $k \in U(m+1)$ such that

$$
\operatorname{Ad}_{k}(Z(\mathbf{e})) \subset \mathfrak{t} .
$$

Proof. It suffices to prove that $Z(\mathbf{e})$ is abelian. To this end let $X, Y \in$ $Z(\mathbf{e})$. By the lemma we may write $X$ as a linear combination of the edges of $\mathbf{e}$. But by definition $Y$ centralizes all the edges of $\mathbf{e}$.

\section{Lemma 4.2.4.}

$$
\operatorname{Im} T_{\mathbf{e}} \mu=\operatorname{Im} T_{e} \mu_{r}+\mathbf{E} .
$$

Proof. Define an action of $\left(\mathbb{R}_{+}\right)^{n}$ on Pol by $\mathbf{a} \cdot \mathbf{e}=\left(a_{1} e_{1}, a_{2} e_{2} \cdots, a_{n} e_{n}\right)$, where $\mathbf{a}=\left(a_{1}, \cdots, a_{n}\right)$. It is immediate that $\tilde{N}_{\mathbf{r}}$ is a cross-section for this action and consequently we have Pol $\cong\left(\mathbb{R}_{+}\right)^{n} \times \widetilde{N}_{\mathbf{r}}$. Now let $\mathbf{e} \in$ Pol. Then

$$
\mu(\mathbf{a} \cdot \mathbf{e})=a_{1} e_{1}+a_{2} e_{2}+\cdots+a_{n} e_{n} .
$$

The lemma follows upon differentiating this identity with respect to e and the action of $\left(\mathbb{R}_{+}\right)^{n}$. 
The following proposition will play a critical role in our analysis of the diagram below.

Proposition 4.2.5. Suppose that $\mathbf{e} \in$ CPol. Then $T_{\mathbf{e}} \mu$ maps onto $\mathcal{H}_{m+1}$.

Proof. We again use the fact that $\left(\operatorname{Im} T_{\mathbf{e}} \mu_{\mathbf{r}}\right)^{\perp}=Z(\mathbf{e})$. By Lemma 4.2.2 the directions coming by changing the side lengths (the action of $\left.\left(\mathbb{R}_{+}\right)^{n}\right)$ contain $Z(\mathbf{e})$. The result now follows from Lemma 4.2.4.

Let $\mathbf{s}:$ Pol $\rightarrow \mathbb{R}^{n}$ and $\overline{\mathbf{s}}:$ CPol $\rightarrow \mathbb{R}^{n}$ denote the side-length maps, and let $\mathbf{j}: \mathrm{CPol} \rightarrow \mathrm{Pol}$ and $\mathbf{k}: \widetilde{N}_{\mathbf{r}} \rightarrow$ Pol be the inclusions. It is immediate (by using the action of $\left(\mathbb{R}_{+}\right)^{n}$ ) that $T_{\mathbf{e}} \mathbf{s}$ maps onto $\mathbb{R}^{n}$.

We will need the subspaces of Pol and CPol obtained by fixing the sums of the side lengths (but not the side lengths themselves). For $\lambda \in \mathbb{R}_{+}$, define

$$
\begin{aligned}
\mathrm{Pol}_{\lambda} & =\left\{\mathbf{e} \in \mathrm{Pol} \mid \sum_{i=1}^{n}\left\|e_{i}\right\|=\lambda\right\} \\
\mathrm{CPol}_{\lambda} & =\mathrm{CPol} \cap \mathrm{Pol}_{\lambda} .
\end{aligned}
$$

We observe that $T_{\mathbf{e}} \mu$ maps $T_{\mathbf{e}} \mathrm{Pol}_{\lambda}$ into $\mathcal{H}_{m+1}^{0}$, and moreover it is an immediate consequence of Proposition 4.2.5 that if $\mathbf{e}$ is closed then this map is onto.

Remark 4.2.6. In what follows we will use the fact that if $f: X \rightarrow Y$ is a real-analytic map of real analytic sets with $f(x)=y$ then the sequence

$$
T_{x}\left(f^{-1}(y)\right) \rightarrow T_{x}(X) \rightarrow T_{y}(Y)
$$

is exact at $T_{x}(X)$ (the second arrow is $\left.T_{x}(f)\right)$.

Theorem 4.2.7. The set of critical values of $\overline{\mathbf{s}}$ is the union of the walls.

Proof. We have seen that $\mathbf{r}$ lies on a wall if, and only if, $\Lambda_{\mathbf{r}} \mathbb{I}$ is a critical value of $\mu_{\mathbf{r}}: \widetilde{N}_{\mathbf{r}} \rightarrow \mathcal{H}_{m+1}$. Let $\mathbf{e} \in \widetilde{M}_{\mathbf{r}}$. The result will follow once we prove that $T_{\mathbf{e}} \mu_{\mathbf{r}}$ is onto if, and only if, $T_{\mathbf{e}} \overline{\mathbf{S}}$ is onto. This follows from a diagram chase in the following commutative diagram. Recall that $\rho=\sum_{i=1}^{n} r_{i}$. Let $\mathbb{R}_{0}^{n}$ denote the subspace of $n$-tuples with sum 0 . It is clear that $T_{\mathbf{e}} \mathbf{s}$ maps $T_{\mathbf{e}} \operatorname{Pol}_{\rho}$ onto $\mathbb{R}_{0}^{n}$. 


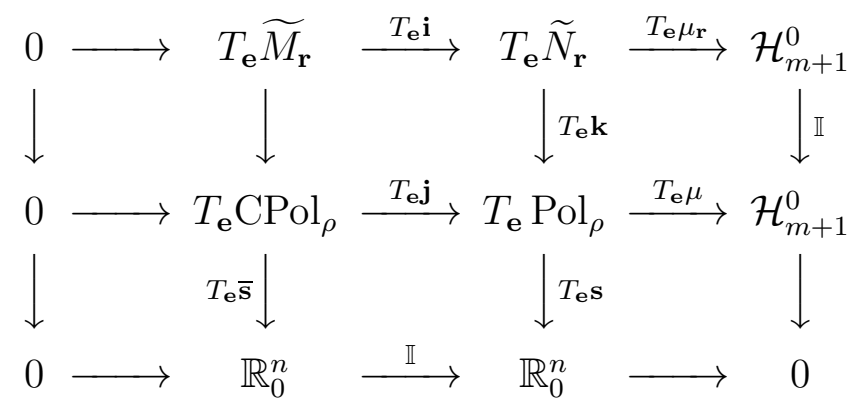

We will perform the diagram chase that proves $T_{\mathbf{e}} \overline{\mathbf{s}}$ onto $\Longrightarrow T_{\mathbf{e}} \mu_{\mathbf{r}}$ onto. To this end let $y \in \mathcal{H}_{m+1}^{0}$. We will construct $x \in T_{\mathbf{e}} \widetilde{N}_{\mathbf{r}}$ with $T_{\mathbf{e}} \mu_{\mathbf{r}}(x)=y$. Indeed, since $T_{\mathbf{e}} \mu$ is onto there exists $z \in T_{\mathbf{e}} \operatorname{Pol}_{\rho}$ with $T_{\mathbf{e}} \mu(z)=y$. By our assumption that $T_{\mathbf{e}} \overline{\mathbf{s}}$ is onto there exists $w \in$ $T_{\mathbf{e}} \mathrm{CPol}_{\rho}$ with $T_{\mathbf{e}} \overline{\mathbf{s}}(w)=T_{\mathbf{e}} \mathbf{s}(z)$. Then $T_{\mathbf{e}} \mathbf{s}\left(z-T_{\mathbf{e}} \mathbf{j}(w)\right)=0$.

Since the next to last vertical sequence is exact at $T_{\mathbf{e}}$ Pol by Remark 4.2.6, there exists $x \in T_{\mathbf{e}} \widetilde{N}_{\mathbf{r}}$ with $T_{\mathbf{e}} \mathbf{k}(x)=z-T_{\mathbf{e}} \mathbf{j}(w)$. Then $T_{\mathbf{e}} \mu_{\mathbf{r}}(x)=$ $y$ as required.

This theorem is a critical first step towards finding the topologies of the moduli spaces $M_{\mathbf{r}}$. We define a chamber of the polyhedral cone $C(n, m+1)$ to be a connected component of the complement of the union of the walls. Then we have the following corollary of the previous theorem.

Corollary 4.2.8. The sidelength map $\mathbf{s}$ is a (trivial) fiber bundle over each chamber of $C(n, m+1)$. Hence if $\mathbf{r}$ and $\mathbf{r}^{\prime}$ lie in the same chamber, the moduli spaces $M_{\mathbf{r}}$ and $M_{\mathbf{r}^{\prime}}$ are diffeomorphic.

Proof. The map $\mathbf{s}$ is a proper submersion over each chamber, hence by the Ehresmann Fibration Theorem, BrJa, pg. 84], it is a fiber bundle (necessarily trivial since chambers are contractible).

In fact we have to replace $\operatorname{Pol}_{\rho}$ by its quotient by $S U(m+1)$ in the argument above, but the reader will check that the critical set of the map induced by $\overline{\mathbf{s}}$ on this quotient remains the same.

Remark 4.2.9. It is possible to implement wall-crossing techniques in order to compute the topologies of the moduli spaces $M_{\mathbf{r}}-$ see[Hu or Goldin for cohomology computations.

\section{Bending Hamiltonians}

Kapovich and Millson ( KM96] studied an integrable Hamiltonian system on $\widetilde{M}_{\mathbf{r}}$ in the case $m=1$ and $e_{2}=-e_{1}$, i.e. in $\mathcal{H}_{2}^{0} \equiv \mathfrak{s u}(2)$, which is isomorphic to Euclidean space $\mathbb{E}^{3}$. In $\S 1$ we introduced the 
diagonals $A_{0}=e_{1}$ and $A_{i}=e_{1}+\cdots+e_{i+1}, i=1, \ldots, n-2$. In this case, a closed polygon has $A_{n-1}=\Lambda \mathbb{I}$. It was shown that the functions $f_{i}(\mathbf{e})=\left\|A_{i}\right\|$ Poisson commute; $\left\|A_{i}\right\|$ is the positive eigenvalue of $A_{i}$. The diagonal $A_{i}$ divides the polygon into two "flaps", and the flow generated by $f_{i}$ is $2 \pi$-periodic, consisting of a rigid rotation of one flap about the diagonal.

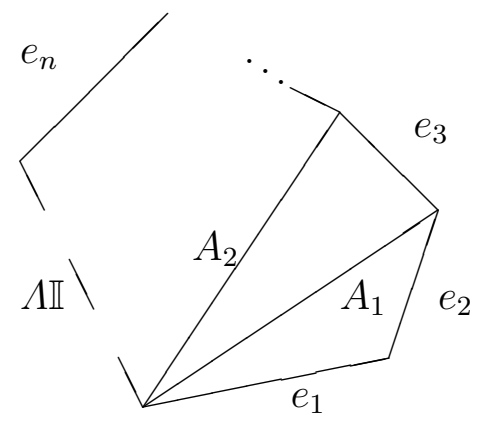

Figure 1. A polygon in $\mathfrak{u}(m+1)$

The analogs of "bending Hamiltonians" for $m>1$ are again the eigenvalues of the diagonals. Now, however, $A_{n-1}=\Lambda \mathbb{I}$, indicated by a dashed line in Figure 1; it would be absent in $\mathfrak{s} u(m+1)$.

Notation 5.1. The eigenvalues of $A_{i}$ are denoted by $\lambda_{i j}$ in decreasing order, $\lambda_{i 1} \geq \ldots \geq \lambda_{i, m+1}$.

We note that $A_{n-2}=\Lambda \mathbb{I}-e_{n}$, has eigenvalues $\Lambda$ (multiplicity $m$ ) and $\Lambda-r_{n}$, and those are fixed. Thus only the $\lambda_{i j}$ for $1 \leq i \leq n-$ 3 are of possible interest. Furthermore, it will be seen in 6.1 that off submanifolds of $\widetilde{M}_{\mathbf{r}}$ of lower dimension, the nontrivial $\lambda_{i j}$ (those not identically 0 or $\Lambda$ ) are simple. In that case, they will be smooth functions of $\mathbf{e}$, which is assumed throughout the present section.

5.1. Bending Flows. We want to calculate the Hamiltonian vector fields and flows generated by the $\lambda_{i j}$. By analogy with the case of $\mathbb{E}^{3}$, we call them "bending flows".

On a product of orbits, the Poisson bracket is the sum of the orbit brackets, and the next formula is evident from (2.1.2): 
Proposition 5.1.1. Suppose $f: \widetilde{M}_{\mathbf{r}} \rightarrow \mathbb{C}$ is smooth and depends only on $e_{1}, \ldots, e_{i+1}$. Then the Hamiltonian system generated by $f$ is

$$
\dot{e}_{k}= \begin{cases}{\left[\nabla_{k} f\left(e_{1}, \ldots, e_{i+1}\right), e_{k}\right],} & \text { if } 1 \leq k \leq i+1, \\ 0, & \text { if } i+1<k \leq n,\end{cases}
$$

where $\nabla_{k}$ denotes gradient with respect to $e_{k}$, all other $e_{j}$ being held fixed.

To solve these equations when $f=\lambda_{i j}$, we recall a standard lemma from perturbation theory.

Lemma 5.1.2. Let $\lambda$ be an isolated eigenvalue of $A \in \mathcal{H}_{m+1}$, with unit eigenvector $u$. Then $\nabla \lambda(A)=\sqrt{-1} u \otimes u^{*}$.

Proof. For $A^{\prime}$ sufficiently close to $A$, the eigenvalue $\lambda\left(A^{\prime}\right)$ and (with proper choice of phase) normalized eigenvector $u\left(A^{\prime}\right)$ vary analytically in a neighborhood of $\lambda, u$. Take a curve $A(s) u(s)=\lambda(s) u(s)$, and take the inner product with the unit length $u(s)$ to get $\lambda(s)=$ $(A(s) u(s), u(s))$. Differentiate and set $s=0$, and use $(A u, \dot{u}(0))+$ $(A \dot{u}(0), u)=\lambda((u, \dot{u}(0))+(\dot{u}(0), u))=0$, resulting in

$$
\dot{\lambda}(0)=(\dot{A}(0) u, u)=\operatorname{Im} \operatorname{Tr}\left(\sqrt{-1} u \otimes u^{*} \dot{A}(0)\right),
$$

as was to be shown.

Write $E_{j}(A)$ for the spectral projection onto the $\lambda_{j}$ eigenspace of $A$; the lemma thus states that $\nabla \lambda_{j}(A)=\sqrt{-1} E_{j}(A)$. We compute the Hamiltonian flows generated by the functions $\lambda_{i j}: \mathbf{e} \mapsto \lambda_{j}\left(A_{i}\right)$ on $\widetilde{N}_{\text {r. }}$. These functions are invariant under the $\mathrm{U}(\mathrm{m}+1)$ action, and hence descend to the symplectic quotient $M_{\mathbf{r}}$. The $\lambda_{i j}$ are smooth when they are simple eigenvalues.

Proposition 5.1.3. For $i=1, \ldots, n-3$ and $j=1, \ldots, m+1, \lambda_{i j}$ is the Hamiltonian for the system

$$
\dot{e}_{k}= \begin{cases}\sqrt{-1}\left[E_{j}\left(e_{1}+\cdots+e_{i+1}\right), e_{k}\right], & \text { if } 1 \leq k \leq i+1 \\ 0, & \text { if } i+1<k \leq n\end{cases}
$$

The Hamiltonian flow $\phi_{i j}^{t}(\mathbf{e})=\mathbf{e}(t)$ is given by

$$
e_{k}(t)= \begin{cases}\left(\operatorname{Ad} \exp \left(\sqrt{-1} t E_{j}\left(A_{i}\right)\right)\right)\left(e_{k}\right), & \text { if } 1 \leq k \leq i+1, \\ e_{k}, & \text { if } i+1<k \leq n\end{cases}
$$

Proof. To obtain the system (5.1.2) we wish to apply Proposition 5.1.1. It is necessary to relate the partial gradients $\nabla_{k} \lambda_{i j}$ to the full gradient, 
$\nabla \lambda_{i j}=\sqrt{-1} E_{j}\left(A_{i}\right)$. According to Lemma 5.1.2, the former are found by computing

$$
\dot{A}_{i}(s)=\left(e_{1}+\cdots+e_{k}(s)+\cdots+e_{i+1}\right)^{\cdot}=\dot{e}_{k}(s),
$$

but because $\dot{A}_{i}(0)$ is tangent to $\mathcal{O}_{r_{k}}$, this only determines $\nabla_{k} \lambda_{i j}$ up to a vector normal to the orbit:

$$
\nabla_{k} \lambda_{i j}\left(A_{i}\right)=\sqrt{-1} E_{j}\left(A_{i}\right)+\xi_{k}, \quad\left[\xi_{k}, e_{k}\right]=0 .
$$

Then $\left[\nabla_{k} \lambda_{i j}, e_{k}\right]=\sqrt{-1}\left[E_{j}\left(A_{i}\right), e_{k}\right]$, and (5.1.2) follows.

Next, add the equations (5.1.2) for $1 \leq k \leq i+1$ to find

$$
\dot{A}_{i}(t)=\sqrt{-1}\left[E_{j}\left(A_{i}(t)\right), A_{i}(t)\right] .
$$

Since $A_{i}$ commutes with its own spectral projections, we get $\dot{A}_{i}(t)=0$ and $A_{i}(t)=A_{i}$. With constant $A_{i}$, the solution of (5.1.2) is immediate.

Corollary 5.1.4. The flows $\phi_{i j}$ have period $2 \pi$ in $t$.

Proof. If $P$ is a projection, then $P^{2}=P$. Consequently, $\exp (\sqrt{-1} t P)=$ $\mathbb{I}+(\exp (\sqrt{-1} t)-1) P$, which has period $2 \pi$.

5.2. Involutivity. It is not a priori clear from the formulas for $\phi_{i j}$ that these flows commute. This is a short calculation; we again work only with simple eigenvalues of the $A_{i}$ on $\widetilde{N}_{\mathbf{r}}$, and the flows will also commute on $M_{\mathbf{r}}$.

Proposition 5.2.1. $\left\{\lambda_{i j}, \lambda_{k \ell}\right\}=0$ for $1 \leq i, k \leq n-3$ and $1 \leq j, \ell \leq$ $m+1$.

Proof. By Proposition 5.1.1 and the proof of Proposition 5.1.3.

$$
\left\{\lambda_{i j}, \lambda_{k \ell}\right\}(\mathbf{e})=\sum_{s=1}^{i+1} \operatorname{Im} \operatorname{Tr}\left(e_{s}\left[\sqrt{-1} E_{j}\left(A_{i}\right)+\xi_{s}, \sqrt{-1} E_{\ell}\left(A_{k}\right)+\eta_{s}\right]\right),
$$

where again $\xi_{s}, \eta_{s}$ commute with $e_{s}$. The ad-invariance of the trace form produces $\left[\xi, e_{s}\right]$ and $\left[\eta, e_{s}\right]$, which are zero. This leaves

$$
\begin{aligned}
\left\{\lambda_{i j}, \lambda_{k \ell}\right\}(\mathbf{e}) & =-\sum_{s=1}^{i+1} \operatorname{Im} \operatorname{Tr}\left(\left[e_{s}, E_{j}\left(A_{i}\right)\right] E_{\ell}\left(A_{k}\right)\right) \\
& =-\operatorname{Im} \operatorname{Tr}\left(\left[A_{i}, E_{j}\left(A_{i}\right)\right] E_{\ell}\left(A_{k}\right)\right) \\
& =0 .
\end{aligned}
$$


Remark 5.2.2. The proof works more generally, if instead of $A_{i}$ and $A_{k}$ one has $\sum_{I} e_{i}$ and $\sum_{J} e_{j}$, with $I \subset J$. Thus, for example, the eigenvalues of $e_{2}+e_{3}$ and $e_{1}+\cdots+e_{5}$ are in involution. On the other hand, if $\lambda, \mu$ are eigenvalues of $e_{1}+e_{2}$ and $e_{2}+e_{3}$, respectively, then

$$
\{\lambda, \mu\}(\mathbf{e})=-\operatorname{Im} \operatorname{Tr}\left(e_{2}\left[E_{\lambda}\left(e_{1}+e_{2}\right), E_{\mu}\left(e_{2}+e_{3}\right)\right]\right),
$$

which need not be zero. See [KM01] for more information.

\section{A Complete Set of Bending Flows}

The eigenvalues $\lambda_{i j}(\mathbf{e})$ have been shown to Poisson commute, and to generate $2 \pi$-periodic flows. If there were $\frac{1}{2} \operatorname{dim} M_{\mathbf{r}}$ eigenvalues and if they were smooth, they would constitute a set of action variables on $M_{\mathbf{r}}$. Smoothness everywhere cannot be achieved, but there are $\frac{1}{2} \operatorname{dim} M_{\mathbf{r}}$ that are smooth and functionally independent on a dense open submanifold of $M_{\mathrm{r}}$. This section presents the proof.

6.1. The Weinstein-Aronszajn Formula. The diagonal $A_{i}$ is a rankone perturbation of $A_{i-1}$, and because of this, the eigenvalues $\lambda_{i j}$ and $\lambda_{i-1, j}$ are related in a special way. This connection is the simplest instance of the Weinstein-Aronszajn formula Kato, Ch.4, §6]. We describe the formula and two consequences that will be used later.

Let $A$ be an $(m+1) \times(m+1)$ Hermitean matrix with eigenvalues $\lambda_{1}, \ldots, \lambda_{m+1}$ and let $u_{1}, \ldots, u_{m+1}$ be corresponding orthonormal eigenvectors. (If an eigenvalue has multiplicity $>1$, which is now permitted, the choice of its eigenvectors is irrelevant). Let $w \in \mathbb{C}^{m+1}$ be a unit vector, and let $r \in \mathbb{R}$. Set $L=A+r w \otimes w^{*}$, and call its eigenvalues $\nu_{1}, \ldots, \nu_{m+1}$. Finally, define $\alpha_{1}, \ldots, \alpha_{m+1} \in \mathbb{C}$ by $w=\sum_{j=1}^{m+1} \alpha_{j} u_{j}$.

\section{Proposition 6.1.1.}

$$
\frac{\operatorname{det}(z \mathbb{I}-L)}{\operatorname{det}(z \mathbb{I}-A)}=1-r \sum_{j=1}^{m+1} \frac{\left|\alpha_{j}\right|^{2}}{z-\lambda_{j}}
$$

Proof. Write $R_{z}=(z \mathbb{I}-A)^{-1}$ for the resolvent of $A$. The left side of (6.1.1) is

$$
\begin{aligned}
& =\operatorname{det}\left((z \mathbb{I}-A)^{-1}\left(z \mathbb{I}-A-r w \otimes w^{*}\right)\right) \\
& =\operatorname{det}\left(\mathbb{I}-R_{z}\left(r w \otimes w^{*}\right)\right) \\
& =\operatorname{det}\left(\mathbb{I}-r\left(R_{z} w\right) \otimes w^{*}\right) .
\end{aligned}
$$

Now, $\operatorname{det}\left(\zeta \mathbb{I}-r\left(R_{z} w\right) \otimes w^{*}\right)$ is the characteristic polynomial of a rankone matrix, and so has an $m$-fold root at $\zeta=0$ and a simple root at 
$\zeta=r\left(R_{z} w, w\right)$. Setting $\zeta=1$ we get

$$
\operatorname{det}\left(\mathbb{I}-r\left(R_{z} w\right) \otimes w^{*}\right)=1-r\left(R_{z} w, w\right) .
$$

The lemma now follows by expanding $w$ in (6.1.2) in the basis $u_{j}$.

It is convenient to write (6.1.1) more explicitly:

$$
\frac{\left(z-\nu_{1}\right) \ldots\left(z-\nu_{m+1}\right)}{\left(z-\lambda_{1}\right) \ldots\left(z-\lambda_{m+1}\right)}=1-r \sum_{j=1}^{m+1} \frac{\left|\alpha_{j}\right|^{2}}{z-\lambda_{j}} .
$$

Corollary 6.1.2. The $\left|\alpha_{j}\right|^{2}$ are rational functions of $\nu_{k}, \lambda_{\ell}, 1 \leq k, \ell \leq$ $m+1$.

Finally, we show that the eigenvalues of $A$ and $L$ interlace. This will play a basic role below.

Proposition 6.1.3. If $r>0$, then $\nu_{1} \geq \lambda_{1} \geq \nu_{2} \cdots \geq \nu_{m+1} \geq \lambda_{m+1}$. If $r<0$, we have $\lambda_{1} \geq \nu_{1} \ldots$ instead.

Proof. Suppose $r>0$. It suffices to prove the proposition for a dense set of $w$, so that we may assume $\left|\alpha_{j}\right|^{2}>0$ for all $j$. Let $R(z)$ be the rational function on the right side of (6.1.3). Since $\lim _{z \rightarrow \infty} R(z)=$ 1 and $\lim _{z \downarrow \lambda_{1}}=-\infty, R$ has a zero in $\left(\lambda_{1}, \infty\right)$. Likewise, because $\lim _{z \uparrow \lambda_{j}}=+\infty$ and $\lim _{z \downarrow \lambda_{j+1}}=-\infty, R$ has a zero in $\left(\lambda_{j+1}, \lambda_{j}\right)$. This provides $m+1$ zeros of $R$, which must coincide with the zeros $\nu_{j}$ of the left side of (6.1.3).

6.2. Gel'fand-Tsetlin Patterns. Let $\mathbf{e} \in \widetilde{M}_{\mathbf{r}}$. We will arrange the eigenvalues of $A_{0}=e_{1}, A_{1}, \ldots, A_{n-1}$ in a triangle with vertex at the bottom. The eigenvalues of $A_{k}$ are written in row $k$ of the triangle, along with some space-filling zeros. For $0 \leq k \leq m$, the rank of $A_{k}$ is at most $k+1$, so zero must be at least an $(m-k)$-fold eigenvalue of $A_{k}$. Those zeros are not recorded. When $k>m$, there are $m+1$ eigenvalues, potentially nonzero; these are recorded along with $k-m$ zeros. Figure 2 shows the case $m=2, n=6$. Note that entries of successive rows are offset to reflect the interlacing property deduced in Proposition 6.1.3. This diagram is called a Gel'fand-Tsetlin pattern, or GTs pattern for short. It is denoted by $\Gamma(\mathbf{e})$. The extra zeros will be explained in $\S 11$, see Remark 8.5 .

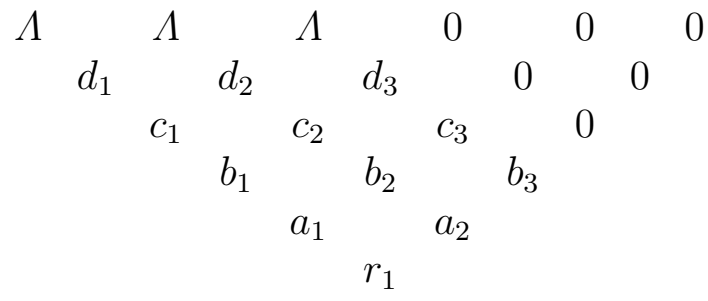


Figure 2

Since $\mathbf{e} \in \widetilde{M}_{\mathbf{r}}$, there are additional restrictions on the entries of $\Gamma(\mathbf{e})$. Row $n-1$ must consist of $m+1 \Lambda$ 's (because $e_{1}+\cdots+e_{n}=\Lambda \mathbb{I}$ ) and $(n-m-1)$ zeros. The interlacing property forces the first $m$ entries of row $n-2$ to be $\Lambda$, so in Figure $2, d_{1}=d_{2}=\Lambda$. Likewise, $c_{1}=\Lambda$. It becomes apparent that the extra zeros remind one that (for example) the eigenvalues $d_{3}=\lambda_{4,3}$ and $c_{3}=\lambda_{3,3}$ must be non-negative.

Moreover,

$$
\operatorname{Tr} A_{k}=\operatorname{Tr}\left(e_{1}+\cdots+e_{k+1}\right)=r_{1}+\cdots+r_{k+1},
$$

which is a linear constraint on the rows of $\Gamma(\mathbf{e})$. In Figure 2, that leaves $c_{2}, b_{1}, b_{2}, a_{1}$ as potentially independent commuting Hamiltonians, and indeed $\operatorname{dim}_{\mathbb{R}} M_{\mathbf{r}}=8$ in this case.

We summarize this discussion.

Definition 6.2.1. Let $m, n, \mathbf{r}$ be fixed. We write $\mathbf{P}$ for the convex polytope of GTs patterns satisfying the following conditions.

(1) There are $n$ rows numbered $0, \ldots, n-1$ (starting at the bottom);

(2) Row $n-1$ consists of $m+1 \Lambda$ 's and $n-m-1$ zeros;

(3) The sum of the entries of row $k$ is $\sum_{i=0}^{k} r_{i+1}$.

(4) The interlacing property $\lambda_{i j} \geq \lambda_{i-1, j} \geq \lambda_{i, j+1}$ holds.

Proposition 6.2.2. $\operatorname{dim} \mathbf{P}=(n-m-2) m=\frac{1}{2} \operatorname{dim}_{\mathbb{R}} M_{\mathbf{r}}$.

Proof. There are two cases: (1) $n \geq 2(m+1)$ and (2) $n \leq 2 m+1$. The difference comes from the position of row $m$, corresponding to the eigenvalues of $A_{m}=e_{1}+\cdots+e_{m+1}$. Generically, this matrix will have full rank. In case (2), some of its eigenvalues are forced, by interlacing, to be $\Lambda$. In case (1), all the automatic $\Lambda$ 's have been "exhausted". (Figure 2 falls into the latter category). Let us sketch the counting.

Case (1): Unconstrained $\lambda_{i j}$ can appear in rows $i=1, \ldots, n-3$. Break this index set into three parts: $S_{1}=\{1, \ldots, m\}, S_{2}=\{m+$ $1, \ldots, n-m-2\}, S_{3}=\{n-m-1, \ldots, n-3\}$. If $n=2(m+1)$ (as in Figure 2), then $S_{2}=\emptyset$. The numbers of unconstrained $\lambda_{i j}$ for the corresponding $A_{k}$ are

- In $S_{1}, 1, \ldots, m$;

- in $S_{2}, m, \ldots, m$;

- in $S_{3}, m-1, \ldots, 1$.

Adding, we obtain

$$
\frac{m(m+1)}{2}+\left(n-(2(m+1)) m+\frac{m(m-1)}{2}=(n-m-2) m .\right.
$$


Case (2): We set $S_{1}=\{1, \ldots, n-m-2\}, S_{2}=\{n-m-1, \ldots, m\}$, $S_{3}=\{m+1, \ldots, n-3\}$ (if $m=1,2$, then $S_{3}=\emptyset$ ). The numbers of unconstrained $\lambda_{i j}$ are:

- In $S_{1}, 1, \ldots, n-m-2$;

- in $S_{2}, n-m-2, \ldots, n-m-2$;

- in $S_{3}, n-m-3, \ldots, 1$.

Now add.

6.3. Constructing a polygon with given GTs pattern. In the last section, we saw that $\Gamma\left(M_{\mathbf{r}}\right) \subset \mathbf{P}$. We now prove the converse.

Theorem 6.3.1. (i) $\Gamma\left(M_{\mathbf{r}}\right)=\mathbf{P}$. (ii) There are $\frac{1}{2} \operatorname{dim} M_{\mathbf{r}}$ functionally independent $\lambda_{i j}$ 's.

Proof. Let $\mathcal{S}_{m+1} \subset \mathcal{H}_{m+1}$ denote the space of real symmetric matrices, and let $\widetilde{M}_{\mathbf{r}}\left(\mathcal{S}_{m+1}\right)$ be the set of polygons in $\widetilde{M}_{\mathbf{r}}$ with each $e_{i} \in \mathcal{S}_{m+1}$. The obvious inclusion $\widetilde{M}_{\mathbf{r}}\left(\mathcal{S}_{m+1}\right) \hookrightarrow \widetilde{M}_{\mathbf{r}}$ is the analog of the inclusion $\widetilde{M}_{\mathbf{r}}\left(\mathbb{R}^{2}\right) \hookrightarrow \widetilde{M}_{\mathbf{r}}\left(\mathbb{R}^{3}\right)$ used in KM96. We will see later that elements of $\mathcal{S}_{m+1}\left(\widetilde{M}_{\mathbf{r}}\right)$ can be thought of as "unbent" polygons; these will be important in our proof of the involutivity of the angle variables in the next section. We now show that

$$
\Gamma\left(\widetilde{M}_{\mathbf{r}}\left(\mathcal{S}_{m+1}\right)\right)=\mathbf{P} .
$$

Since $\Gamma: \widetilde{M}_{\mathbf{r}}\left(\mathcal{S}_{m+1}\right) \rightarrow \mathbf{P}$ is continuous (though not differentiable), the image of $\Gamma$ is closed, and it suffices to prove that the image of $\Gamma$ contains the interior $\mathbf{P}^{o}$ of $\mathbf{P}$. Thus, choose a GTs pattern $\gamma$ in which all unconstrained inequalities are strict; we are to find e $\in \Gamma\left(\widetilde{M}_{\mathbf{r}}\left(\mathcal{S}_{m+1}\right)\right)$ such that $\Gamma(\mathbf{e})=\gamma$.

Set $A_{0}=r_{1} w_{1} \otimes w_{1}^{*}$, where $w_{1}$ is an arbitrary real unit vector. Assuming that a real symmetric $A_{k-1}$ with a given spectrum has been found, we want $w_{k+1} \in \mathbb{R}^{m+1}$ so that

$$
A_{k}=A_{k-1}+r_{k+1} w_{k+1} \otimes w_{k+1}^{*}
$$

has the required next spectrum.

We carry out the induction step for Case (1), in the terminology of Proposition 6.2.2. First, let $k \in S_{1}$. Thus

$$
A_{k-1}=\sum_{j=1}^{k} r_{j} w_{j} \otimes w_{j}^{*} ;
$$

it has spectrum $\left\{\lambda_{1}, \ldots, \lambda_{k}, 0, \ldots, 0\right\}$ with $\lambda_{1}>\cdots>\lambda_{k}>0$, and $\sum_{i=1}^{k} \lambda_{i}=\sum_{i=1}^{k} r_{i}$. We are further given $\nu_{i}$ with

$$
\nu_{1}>\lambda_{1}>\nu_{2}>\cdots>\lambda_{k}>v_{k+1}>0
$$


and $\sum_{i=1}^{k+1} \nu_{i}=\sum_{i=1}^{k+1} r_{i}$.

Let $u_{1}, \ldots, u_{k}, u$ be normalized (real) eigenvectors of $A_{k-1}$ corresponding to $\lambda_{1}, \ldots, \lambda_{k}, 0$, and seek $w_{k+1}$ in the form

$$
w_{k+1}=\sum_{j=1}^{k} \alpha_{j} u_{j}+\alpha u
$$

with $\alpha_{i}, \alpha$ real.

Now solve for $\left|\alpha_{j}\right|^{2}, 1 \leq j \leq k$ and $|\alpha|^{2}$ in equation (6.1.3), which takes the special form

$$
\frac{\left(z-\nu_{1}\right) \ldots\left(z-\nu_{k+1}\right) z^{m-k}}{\left(z-\lambda_{1}\right) \ldots\left(z-\lambda_{k}\right) z^{m-k+1}}=1-r_{k+1}\left(\sum_{j=1}^{k} \frac{\left|\alpha_{j}\right|^{2}}{z-\lambda_{j}}+\frac{|\alpha|^{2}}{z}\right) .
$$

Clearly one can take $\alpha_{i}, \alpha$ real. Taking traces in equation (6.3.2), we get

$$
\sum_{j=1}^{k+1} r_{j}=\sum_{j=1}^{k+1} \nu_{j}=\sum_{j=1}^{k} r_{j}+r_{k+1}\left\|w_{k+1}\right\|^{2}
$$

whence $\left\|w_{k+1}\right\|=1$.

The same procedure works in the remaining subcases as well; for $k \in S_{2}$ the eigenvalues $\lambda_{j}$ and $\nu_{j}$ are simple, while for $k \in S_{3}$, account must be taken of the multiplicity of $\Lambda$.

Remark 6.3.2. The proof shows that, if $w_{k+1}$ is not required to be real, each term $\alpha_{j} u_{j}$ is determined only up to a multiple $\exp \left(\sqrt{-1} \theta_{k+1, j}\right)$. Thus, the possible polygons e corresponding to a given pattern $\gamma$ lie on a torus. The angle coordinates are studied in the next section.

We conclude by making a choice of functionally independent action variables.

Definition 6.3.3. Let $\mathcal{I}$ be the set of pairs $(i, j)$ satisfying $1 \leq i \leq$ $n-1,1 \leq j \leq i$ which index eigenvalues $\lambda_{i j}$ such that $\lambda_{i j}$ is not forced to be 0 or $\Lambda$, with the further property that $\lambda_{i, j+1}$ is not forced to be 0 (this last condition says that in each row we throw away the right-most $j$ such that $\lambda_{i j}$ is not forced to be 0).

Corollary 6.3.4. The set $\mathcal{I}$ indexes a functionally independent set of action variables $\lambda_{i j}$.

Proof. Indeed, these action variables map onto a polyhedron of dimension equal to the cardinality of $\mathcal{I}$. 
Remark 6.3.5. For general coadjoint orbits, one can define a complete set of constants of motion that reduce to the $\alpha_{j}$ in the rank one case; the construction also makes use of Gel'fand-Tsetlin patterns. Action variables which generate $2 \pi$-periodic flows are not known, however.

\section{Angle Variables and Four-Point Functions}

In this section, we construct angle variables $\theta_{i j}$ conjugate to the action variables $\lambda_{i j}$ discussed thus far. The angles are implicit in Corollary 5.1.4 and Remark 6.3.2, what we now find is a global description.

7.1. Four-point functions and polygons. The geometric picture in KM96] serves as model. For the moment, think of the sides $e_{j}$ as vectors in $\mathbb{R}^{3}$. The action variables are the lengths of the diagonals $A_{i}=e_{1}+\ldots+e_{i+1}$ of the polygon. The corresponding conjugate angle is the oriented dihedral angle between the two triangles spanned, respectively, by $A_{i-1}, e_{i+1}, A_{i}$ and $A_{i}, e_{i+2}, A_{i+1}$. By this we mean the oriented angle between the two normal vectors to the triangles. These two vectors are elements of the plane orthogonal to $A_{i}$. We orient this plane so that a positively oriented basis for the plane followed by $A_{i}$ is a positively oriented basis for $\mathbb{R}^{3}$.

Remark 7.1.1. In an oriented plane $\Pi$ equipped with a positive definite inner product $U \cdot V$, we can define the oriented angle $\angle(U, V)$ for a pair of vectors $U$ and $V$ in $\Pi$ as follows. First we say that two unit vectors $U, V$ make an angle of ninety degrees if $U \cdot V=0$ and the basis $\{U, V\}$ is positively oriented. We let $J$ be the operation of rotation by ninety degrees. We make $\Pi$ into a complex vector space by defining $\imath V:=J V$. Then the unit circle in $\mathbb{C}$ acts simply-transitively on the oriented lines in $\Pi$. We define $\angle(U, V)=\theta$ if $\exp (\imath \theta) U$ is a positive real multiple of $V$. If $\theta=\angle(U, V)$ then we have

$$
\begin{aligned}
& \cos \theta=\frac{U \cdot V}{\|U\|\|V\|} \\
& \sin \theta=\frac{J U \cdot V}{\|U\|\|V\|}
\end{aligned}
$$

For the case at hand, the oriented angle $\theta_{i}$ is given by

$$
\begin{aligned}
\cos \theta_{i} & =\frac{\left(A_{i} \times e_{i+1}\right) \cdot\left(A_{i} \times e_{i+2}\right)}{\left\|A_{i} \times e_{i+1}\right\|\left\|A_{i} \times e_{i+2}\right\|} \\
\sin \theta_{i} & =\frac{\left(A_{i} \times e_{i+1}\right) \times\left(A_{i} \times e_{i+2}\right) \cdot A_{i}}{\left\|A_{i} \times e_{i+1}\right\|\left\|A_{i} \times e_{i+2}\right\|\left\|A_{i}\right\|} .
\end{aligned}
$$


Note that $\theta_{i}=0$ when the triangles are coplanar, so that the collection of planar polygons forms a reference cross-section for the angle variables.

We now transfer (7.1.1) and (17.1.2) back to our Lie algebra $\mathcal{H}_{2}^{0}$ of tracefree Hermitean $2 \times 2$ matrices. Define $f: \mathbb{R}^{3} \rightarrow \mathcal{H}_{2}^{0}$ by

$$
f: \mathbf{x}=\left(x_{1}, x_{2}, x_{3}\right) \mapsto \hat{\mathbf{x}}=\frac{1}{2}\left(\begin{array}{cc}
x_{1} & x_{2}+\sqrt{-1} x_{3} \\
x_{2}-\sqrt{-1} x_{3} & -x_{1}
\end{array}\right)
$$

Then $\widehat{\mathbf{x} \times \mathbf{y}}=\sqrt{-1}[\hat{\mathbf{x}}, \hat{\mathbf{y}}], \mathbf{x} \cdot \mathbf{y}=2 \operatorname{Tr} \hat{\mathbf{x}} \hat{\mathbf{y}}$, and a vector in the $x_{3}=0$ plane corresponds to a real symmetric matrix. (Thus, a planar polygon is represented by a symmetric matrix, cf. Theorem 6.3.1).

We return to identifying vectors with matrices via (7.1.3).

Let $\lambda>0$ and $-\lambda$ be the eigenvalues of $A_{i}$, with orthonormal eigenvectors $u, v$, so that $A_{i}=\lambda\left(u \otimes u^{*}-v \otimes v^{*}\right)$. Write, for notational simplicity,

$$
e_{i+1}=r_{1} w_{1} \otimes w_{1}^{*}-\left(r_{1} / 2\right) \mathbb{I}, \quad e_{i+2}=r_{2} w_{2} \otimes w_{2}^{*}-\left(r_{2} / 2\right) \mathbb{I} .
$$

Then the numerator of (17.1.1) becomes (since $\mathbb{I}$ does not contribute)

$$
2 \operatorname{Tr}\left(\sqrt{-1}\left[A_{i}, r_{1} w_{1} \otimes w_{1}^{*}\right] \sqrt{-1}\left[A_{i}, r_{2} w_{2} \otimes w_{2}^{*}\right]\right),
$$

and the numerator of (7.1.2) becomes

$$
2\left\|A_{i}\right\| \operatorname{Tr}\left(\sqrt{-1} A_{i}\left[r_{1} w_{1} \otimes w_{1}^{*}, r_{2} w_{2} \otimes w_{2}^{*}\right]\right) .
$$

Definition 7.1.2 ([BeSch $]$ ). Let $a, b, c, d \in \mathbb{C}^{m+1}$. Define the fourpoint function by

$$
F_{4}(a, b, c, d)=\frac{(a, b)(b, c)(c, d)(d, a)}{\|a\|^{2}\|b\|^{2}\|c\|^{2}\|d\|^{2}}
$$

where $(\cdot, \cdot)$ is the usual Hermitean inner product.

Two properties of $F_{4}$ are important:

(1) $F_{4}(a, b, c, d)$ may be thought of as function on $\left(\mathbb{C P}^{m}\right)^{4}$; in particular, $F_{4}$ is independent of the phases of its arguments.

(2) $\overline{F_{4}(a, b, c, d)}=F_{4}(a, d, c, b)$ (plus other such symmetries).

A longish calculation, using property (2), gives the following.

Proposition 7.1.3. Expression (7.1.4) reduces to

$$
16 \lambda^{2} r_{1} r_{2} \operatorname{Re} F_{4}\left(w_{1}, u, w_{2}, v\right) .
$$

Expression (7.1.5) reduces to

$$
16 \lambda^{2} r_{1} r_{2} \operatorname{Im} F_{4}\left(w_{1}, u, w_{2}, v\right) .
$$

The denominator in (7.1.1) and (7.1.2) becomes

$$
16 \lambda^{2} r_{1} r_{2}\left|F_{4}\left(w_{1}, u, w_{2}, v\right)\right|
$$


Thus, the oriented dihedral angle is $\theta=\arg F_{4}\left(w_{1}, u, w_{2}, v\right)$.

This formula, suitably adapted, will be shown to define the conjugate angles in the more general case as well.

We mention, as an aside, that the argument of the four-point function has an interesting geometric description.

Theorem 7.1.4. Let $a_{j}, j=1, \ldots, 4$ be four points in $\mathbb{C}^{m+1}$ defining points $p_{j} \in \mathbb{C P}^{m}$. Construct a geodesic quadrilateral $\pi$ in $\mathbb{C P}^{m}$ with vertices at the $p_{j}$. Let $\sigma$ be a two-chain with boundary $\pi$ and let $\omega$ be the Kähler form on $\mathbb{C P}^{m}$. Then

$$
\arg F_{4}\left(a_{1}, a_{2}, a_{3}, a_{4}\right)=-\int_{\sigma} \omega .
$$

Proof. Draw a geodesic segment (a diagonal of the quadrilateral) from $p_{1}$ to $p_{3}$. The analogue of (7.1.6) for triangles was proved in [HM], see also [Go, Ch. 7]. Now choose $\sigma$ to be the union of two two-chains each of which has as boundary one of the two triangles created by drawing the diagonal $p_{1} p_{3}$. Combining (7.1.6) for the triangles gives the equation for the quadrilateral.

7.2. Construction of angle variables. We will define the angle variables as in Proposition 7.1.3. via the four-point function of the w's associated with two consecutive edges and eigenvectors of the diagonal between them. These vectors all involve a choice of phase, and the first goal will be to remove the ambiguity.

Let $M_{\mathbf{r}}^{0}$ be the open subset of $M_{\mathbf{r}}$ on which the interlacing inequalities $\lambda_{i j}>\lambda_{i-1, j}>\lambda_{i, j+1}$ are strict, and let $\widetilde{M}_{\mathbf{r}}^{0}$ be its inverse image in $\widetilde{M}_{\mathbf{r}}$. We consider only polygons in $\widetilde{M}_{\mathbf{r}}^{0}$, so that the (unconstrained) eigenvalues and eigenvectors may be taken to be locally smooth functions of $\mathbf{e}$.

Let $\phi^{t}$ be one of the $\lambda_{i k}$-flows defined in Proposition 5.1.3. We will follow the transformed polygon $\phi^{t}(\mathbf{e})$. Its $\ell$-th edge, $r_{\ell} w_{\ell}^{t} \otimes\left(w_{\ell}^{t}\right)^{*}$, and the normalized $\lambda_{i j}$-eigenvector, $u_{i j}^{t}$, of the diagonal $\phi^{t}\left(A_{i}\right)$, will depend on time $t$. They may be taken to be locally smooth on $M_{\mathbf{r}}^{0}$, but will depend on an initial choice, while the polygon $\phi^{t}(\mathbf{e})$ itself is well defined.

Definition 7.2.1. Make smooth local choices of $w_{\ell}$ and $u_{i j}$. Here $u_{i j}$ is a (choice of) unit length eigenvector belonging to the eigenvalue $\lambda_{j}$ of $A_{i}$. Define

$\alpha_{i j}: \widetilde{M}_{\mathbf{r}}^{0} \rightarrow \mathbb{C},(i, j) \in \mathcal{I}$, by $\alpha_{i j}: \mathbf{e} \mapsto\left(w_{i+1}(\mathbf{e}), u_{i j}(\mathbf{e})\right)\left(u_{i j}(\mathbf{e}), w_{i+2}(\mathbf{e})\right) ;$ 
this depends on the phases of $w_{i+1}, w_{i+2}$. (We will usually drop the argument $\mathbf{e})$. Set

$$
\beta_{i j}=F_{4}\left(w_{i+1}, u_{i j}, w_{i+2}, u_{i, j+1}\right)=\alpha_{i j} \overline{\alpha_{i, j+1}} .
$$

The $\beta_{i j}$ are independent of all phase choices. Finally, we define the angle variables $\theta_{i j},(i, j) \in \mathcal{I}$, by

$$
\theta_{i j}=\arg \beta_{i j}
$$

Clearly the number of four-point functions $\beta_{i j}$ is the same as the number of independent, unconstrained $\lambda_{i j}$ 's, since for every $i$ there is one more $\lambda_{i j}$ than $\beta_{i j}$ and there are no $\beta_{i j}$ 's corresponding to the eigenvalues 0 and $\Lambda$. Thus we obtain the correct formal count of angle variables. We now prove that the angle variables are well-defined on $M_{\mathbf{r}}^{0}$.

\section{Lemma 7.2.2.}

(1) All $\left|\alpha_{i j}\right|^{2}$ are constant under all bending flows $\phi_{k \ell}$.

(2) All $\left|\alpha_{i j}\right|^{2}$ are nonzero on $\widetilde{M}_{\mathbf{r}}^{0}$.

In particular, $\arg \beta_{i j}=\arg \alpha_{i j} \overline{\alpha_{i, j+1}}$ is defined.

Proof. The first statement follows from Proposition 6.1.1 and Corollary 6.1.2. Indeed,

$$
A_{i-1}=A_{i}-r_{i+1} w_{i+1} \otimes w_{i+1}^{*} .
$$

Hence $\left|\left(w_{i+1}, u_{i j}\right)\right|^{2}$, being a rational function of action variables, is a constant of motion. Likewise,

$$
A_{i+1}=A_{i}+r_{i+2} w_{i+2} \otimes w_{i+2}^{*}
$$

implies that $\left|\left(w_{i+2}, u_{i j}\right)\right|^{2}$ is a constant of motion.

To prove the second statement we apply the Weinstein-Aronszajn formula to obtain

$$
\frac{\operatorname{det}\left(z \mathbb{I}-A_{i-1}\right)}{\operatorname{det}\left(z \mathbb{I}-A_{i}\right)}=1+r_{i+1} \sum_{j=1}^{m+1} \frac{\left|\left(w_{i+1}, u_{i j}\right)\right|^{2}}{z-\lambda_{i j}} .
$$

If $\left|\alpha_{i j}\right|=0$ then either $\left|\left(w_{i+1}, u_{i j}\right)\right|=0$ or $\left|\left(w_{i+2}, u_{i j}\right)\right|=0$. Assume first that $\left|\left(w_{i+1}, u_{i j}\right)\right|=0$. ¿From the Weinstein-Aronszajn formula we see that if follows that $\lambda_{i j}$ is not a pole, so the $\left(z-\lambda_{i j}\right)$ in the denominator of the left-hand side must cancel with one of the terms in the numerator. Hence one of the interlacing inequalities between the $i^{\text {th }}$ and $(i-1)^{\text {st }}$ rows is not strict, contradicting the assumption that $\mathbf{e} \in \widetilde{M}_{\mathbf{r}}^{0}$. Similarly, $\left(w_{i+2}, u_{i j}\right) \neq 0$.

Lemma 7.2.3. The $\beta_{i j}$ are invariant under conjugation. 
Proof. Let $g \in \mathrm{U}(\mathrm{m}+1)$ and consider the conjugated polygon $g \mathbf{e} g^{-1}$. Its $\ell^{\text {th }}$ edge is $r_{\ell}\left(g w_{\ell}\right) \otimes\left(g w_{\ell}\right)^{*}$. However, the choice $w_{\ell}\left(g \mathbf{e} g^{-1}\right)$ made in Definition 7.2.1 may not coincide with $g w_{\ell}$. If they differ, it is by a multiple of modulus one. The four-point function $\beta_{i j}$ is not affected by such a factor.

In the following we will make essential use of

Remark 7.2.4. In view of the proof of Lemma 7.2.3, we may replace $w_{\ell}\left(g \mathbf{e} g^{-1}\right)$ by $g w_{\ell}$ in calculations involving $\beta_{i j}$, and for the same reason, $u_{i j}\left(g \mathbf{e} g^{-1}\right)$ by $g u_{i j}$.

We will now compute the Poisson brackets of the action variables with the angle variables.

Lemma 7.2.5.

$$
\left\{\lambda_{i l}, \theta_{i j}\right\}=\left\{\begin{aligned}
1, l & =j \\
-1, l & =j+1 \\
0, l & \neq j, j+1
\end{aligned}\right.
$$

Proof. We will verify, using (5.1.3), that

$$
\beta_{i j}\left(\phi_{i l}^{t}(\mathbf{e})\right)=\left\{\begin{aligned}
\beta_{i j}(\mathbf{e}), & l \neq j, j+1, \\
\exp (\sqrt{-1} t) \beta_{i j}(\mathbf{e}), & l=j, \\
\exp (-\sqrt{-1} t) \beta_{i j}(\mathbf{e}), & l=j+1 .
\end{aligned}\right.
$$

Note from (5.1.3) that the $i^{\text {th }}$ diagonal $A_{i}$ of $\mathbf{e}$ and the $(i+2)$-nd edge are fixed under $\phi_{i l}^{t}$. Hence the normalized eigenvectors $u_{i j}$ of $A_{i}$ are also fixed. Now abbreviate $g_{t}=\exp \left(\sqrt{-1} t E_{l}\left(A_{i}\right)\right)$, and as explained in Remark 7.2.3 make the replacement

$$
w_{i+1}\left(\phi_{i l}^{t}(\mathbf{e})\right)=w_{i+1}\left(g_{t} \mathbf{e} g_{t}^{-1}\right) \rightsquigarrow g_{t} w_{i+1}(\mathbf{e}) .
$$

We obtain

$$
\begin{aligned}
\beta_{i j}\left(\phi_{i l}^{t}(\mathbf{e})\right) & =\left(g_{t} w_{i+1}, u_{i j}\right)\left(u_{i j}, w_{i+2}\right)\left(w_{i+2}, u_{i, j+1}\right)\left(u_{i, j+1}, g_{t} w_{i+1}\right) \\
& =\left(w_{i+1}, g_{t}^{-1} u_{i j}\right)\left(u_{i j}, w_{i+2}\right)\left(w_{i+2}, u_{i, j+1}\right)\left(g_{t}^{-1} u_{i, j+1}, w_{i+1}\right) .
\end{aligned}
$$

Since $E_{l}\left(A_{i}\right) u_{i j}=\delta_{j l} u_{i j}$ the lemma follows by definition of $g_{t}$.

\section{Lemma 7.2.6.}

$$
\left\{\lambda_{i j}, \theta_{k l}\right\}=0, i \neq k .
$$

Proof. If $i<k$ then the $k^{\text {th }}$ diagonal, the $(k+1)$-st edge, and the $(k+2)$-nd edge are fixed by the bending flow $\phi_{i j}^{t}$, and hence $\theta_{k l}$ is unchanged. 
If $i>k$, then the $k^{\text {th }}$ diagonal,the $(k+1)$-st edge and the $(k+2)$-nd edge are rigidly moved by the $g_{t}$ under the bending flow $\phi_{i j}^{t}$, and hence $\theta_{k l}$ is unchanged. (Note that Remark [7.2.3] is used once more).

To remove the redundancy in the $\lambda_{i j}$, we define new action variables $\mu_{i j}$ by the formula

$$
\mu_{i j}=\sum_{k=1}^{j} \lambda_{i k}
$$

As a consequence of the two preceding lemmas we obtain

Proposition 7.2.7. The action variables $\left\{\mu_{i j}\right\}$ and the angle variables $\left\{\theta_{i j}\right\}$ are conjugate

$$
\left\{\mu_{i j}, \theta_{k l}\right\}=\left\{\begin{array}{l}
1, i=k, j=l \\
0, \text { otherwise. }
\end{array}\right.
$$

We deduce two corollaries.

Corollary 7.2.8. The angle variables are functionally independent.

Corollary 7.2.9. The Hamiltonian flows of the new action variables $\left\{\mu_{i j}\right\}$ permute the simultaneous level sets $\left\{\theta_{i j}=c_{i j},(i, j) \in \mathcal{I}\right\}$ transitively.

We now begin the proof that

$$
\left\{\theta_{i j}, \theta_{k l}\right\}=0
$$

Recall that $\mathcal{S}_{m+1}$ is the space of real symmetric $(m+1) \times(m+1)$ matrices. Let $\sigma: \mathcal{H}_{m+1} \rightarrow \mathcal{H}_{m+1}$ be complex conjugation. Then $\mathcal{S}_{m+1}$ is the fixed subspace of $\sigma$. The following lemma is immediate from (2.1.1):

Lemma 7.2.10. The involution $\sigma$ is anti-Poisson (a Poisson isomorphism from $\mathcal{H}_{m+1}$ equipped with the Lie Poisson tensor to $\mathcal{H}_{m+1}$ equipped with the negative of the Lie Poisson tensor).

We obtain

Corollary 7.2.11. If $f$ and $g$ are constant on $\mathcal{S}_{m+1}$, then $\{f, g\}$ vanishes on $\mathcal{S}_{m+1}$.

Proof. Let $\pi(.,$.$) be the Lie Poisson bivector considered as a skew-$ symmetric bilinear form on the cotangent bundle of $\mathcal{H}_{m+1}$. For $x \in$ $\mathcal{S}_{m+1}$ and $u, v$ cotangent vectors at $x$, the Lemma gives $\pi_{x}(u, v)=$ $-\pi_{x}(\sigma u, \sigma v)$. If $u$ and $v$ are conormal covectors at $x$ then they are in the $(-1)$-eigenspace for $\sigma$, and therefore $\pi_{x}(u, v)=0$. But if $f$ and $g$ are constant on $\mathcal{S}_{m+1}$, then $d f_{x}$ and $d g_{x}$ are conormal at $x$. 
As an immediate consequence we have

Lemma 7.2.12. If $f$ and $g$ are constant on $M_{\mathbf{r}}\left(\mathcal{S}_{m+1}\right)$, then $\{f, g\}$ vanishes on $M_{\mathbf{r}}\left(\mathcal{S}_{m+1}\right)$.

Our next goal is to prove that the simultaneous zero level set of the angle variables is $M_{\mathbf{r}}\left(\mathcal{S}_{m+1}\right)$. In order to obtain this we will need two technical lemmas to handle the regions $S_{1}$ and $S_{3}$ (in the notation of Proposition 6.2.2). The first lemma will be used to deal with the region $S_{3}$.

Lemma 7.2.13. Let $V_{i}=\operatorname{ker}\left(A_{i}-\Lambda \mathbb{I}\right), n-m-2 \leq i \leq n-1$. Then

$$
V_{n-1} \supset V_{n-2} \supset \cdots \supset V_{n-m-2}=\{0\} .
$$

Moreover (recalling $A_{i}=A_{i-1}+r_{i+1} w_{i+1} \otimes w_{i+1}^{*}$ ) we have

$$
V_{i-1}=\left\{v \in V_{i}:\left(v, w_{i+1}\right)=0\right\} .
$$

Proof. Let $v \in V_{i-1}$ and $\|v\|=1$. Then

$$
\Lambda=\left(A_{i-1} v, v\right)=\left(A_{i} v, v\right)-r_{i+1}\left|\left(w_{i+1}, v\right)\right|^{2} .
$$

But $\Lambda$ is the largest eigenvalue of $A_{i}$ so $\left(A_{i} v, v\right) \leq \Lambda$. Hence the above equation can hold if and only if

$$
\left(A_{i} v, v\right)=\Lambda \quad\left(\text { so } v \in V_{i}\right) \text { and }\left(w_{i+1}, v\right)=0 .
$$

Corollary 7.2.14. Let $w_{i+1}^{\Lambda}$ be the orthogonal projection of $w_{i+1}$ on the 1 -eigenspace of $A_{i-1}$. Then

$$
w_{i+1}^{\Lambda}=0 .
$$

The next lemma will be used to deal with the region $S_{1}$.

Lemma 7.2.15. Let $U_{i}=\operatorname{ker} A_{i}, 1 \leq i \leq m$. Then

$$
U_{1} \supset U_{2} \supset \cdots \supset U_{m}=\{0\} .
$$

Moreover

$$
U_{i}=\left\{u \in U_{i-1}:\left(u, w_{i+1}\right)=0\right\} .
$$

Proof. Suppose $A_{i} u=0$. Then

$$
0=\left(A_{i} u, u\right)=\left(A_{i-1} u, u\right)+r_{i+1}\left|\left(w_{i+1}, u\right)\right|^{2} .
$$

But $A_{i-1}$ is positive semidefinite and $r_{i+1}>0$. Hence $u \in \operatorname{ker} A_{i-1}$ and $\left(u, w_{i+1}\right)=0$.

Corollary 7.2.16. Let $w_{i+1}^{0}$ be the projection of $w_{i+1}$ on $\operatorname{ker} A_{i}$. Then

$$
w_{i+1}^{0}=0 \text {. }
$$


Now we can prove the result we need. Let $Z(\Theta)$ be the simultaneous zero level set of the angle variables $\left\{\theta_{i j}\right\}$.

\section{Proposition 7.2.17.}

$$
Z(\Theta)=M_{\mathbf{r}}\left(\mathcal{S}_{m+1}\right)
$$

Proof. The inclusion

$$
M_{\mathbf{r}}\left(\mathcal{S}_{m+1}\right) \subset Z(\Theta)
$$

is obvious (all the edges and diagonals are real, so the eigenvectors are real, so the $\beta_{i j}$ are real). The point is to prove the reverse inclusion. We will assume $n \geq 2(m+1)$ and leave the case $n \leq 2 m+1$, which is similar, to the reader.

Given a polygon e with all $\theta_{i j}=0$. We wish to show that a sequence of conjugations of $\mathbf{e}$ by elements of $\mathrm{U}(\mathrm{m}+1)$ will make all sides $e_{k}$ real symmetric, or equivalently, all the $w_{k}$ real. The proof is by descending induction, starting with the last diagonal $A_{n-1}=e_{1}+\cdots+e_{n}=\Lambda \mathbb{I}$, which is of course real symmetric. First, conjugate e by $g \in \mathrm{U}(\mathrm{m}+1)$ (without changing $A_{n-1}$ ) to arrange that $A_{n-2}$ is diagonal, hence real. This moves all the $w_{k}$ to $g w_{k}$, but in the sequel we do not need to keep track of those changes. Now we know that $A_{n-3}$ has the form

$$
A_{n-3}=A_{n-2}-r_{n-1} w_{n-1} \otimes w_{n-1}^{*},
$$

and we want to show that we can move $w_{n-1}$ to a real vector. We have

$$
\operatorname{ker}\left(A_{n-2}-\Lambda \mathbb{I}\right)=\left\{\epsilon_{1}, \ldots, \epsilon_{m}\right\},
$$

where $\left\{\epsilon_{1}, \ldots, \epsilon_{m+1}\right\}$ is the standard basis for $\mathbb{C}^{m+1}$. Suppose $A_{n-2} \epsilon_{m+1}=$ $\mu \epsilon_{m+1}, \mu=\Lambda-r_{n}$.

Write $w_{n-1}$ in the form $w_{n-1}=w_{n-1}^{\Lambda}+w_{n-1}^{\perp}$, where $w_{n-1}^{\Lambda}$ is the orthogonal projection of $w_{n-1}$ onto $\operatorname{ker}\left(A_{n-2}-\Lambda \mathrm{II}\right)$. Hence there exists $z \in \mathbb{C}$ such that $w_{n-1}^{\perp}=z \epsilon_{m+1}$. Since $w_{n-1}$ is defined only up to a complex multiple of unit length, we may multiply $w_{n-1}$ by an element of $S^{1}$ in order to arrange that $z$ be real. Let $c=\left\|w_{n-1}^{\Lambda}\right\|$. Now choose $g \in$ $\mathrm{U}(\mathrm{m}+1)$ such that $g \epsilon_{m+1}=\epsilon_{m+1}$ and $g w_{n-1}^{\Lambda}=c \epsilon_{m}$. Then $g A_{n-2} g^{-1}=$ $A_{n-2}$ (because $g \epsilon_{m+1}=\epsilon_{m+1}$ and $\left.g w_{n-1}=c \epsilon_{m}+z \epsilon_{m+1}\right)$. We change $\mathbf{e}=\left(e_{1}, \ldots, e_{n}\right)$ to $g \mathbf{e} g^{-1}=\left(g e_{1} g^{-1}, \ldots, g e_{n} g^{-1}\right)$.

Next, we show how to find a conjugation $g \mathbf{e} g^{-1}$ that keeps $A_{n-2}, A_{n-3}$ and $w_{n-1}$ real and also makes $g w_{n-2}$ real. This step exhibits the general pattern.

By Lemma 7.2 .13 ,

$$
\begin{aligned}
\operatorname{ker}\left(A_{n-3}-\Lambda \mathbb{I}\right) & =\left\{v \in \operatorname{ker}\left(A_{n-2}-\Lambda \mathbb{I}\right):\left(v, w_{n-1}\right)=0\right\} \\
& =\operatorname{span}\left\{\epsilon_{1}, \ldots, \epsilon_{m-1}\right\} .
\end{aligned}
$$


The matrix $A_{n-3}$ has two new eigenvalues (in addition to $\Lambda$ ); let their eigenvectors be $u_{n-3, m+1}, u_{n-3, m}$. There is one angle variable

$$
\begin{aligned}
\theta_{n-3, m+1}= & \arg \left[\left(w_{n-2}, u_{n-3, m}\right)\left(u_{n-3, m}, w_{n-1}\right)\right. \\
& \left.\left(w_{n-1}, u_{n-3, m+1}\right)\left(u_{n-3, m+1}, w_{n-2}\right)\right]
\end{aligned}
$$

We have seen that $A_{n-3}$ is real symmetric, hence $u_{n-3, j}$ can be chosen to be real for all $1 \leq j \leq m+1$. Since $w_{n-1}$ is real, we may normalize $u_{n-3, m}$ and $u_{n-3, m+1}$ so that $\left(w_{n-1}, u_{n-3, m}\right)>0$ and $\left(w_{n-1}, u_{n-3, m+1}\right)>$ 0 . Since, by assumption, $\theta_{n-3, m+1}=0$, we have

$$
\arg \left(w_{n-2}, u_{n-3, m+1}\right)=\arg \left(w_{n-2}, u_{n-3, m}\right) .
$$

Hence by multiplying $w_{n-2}$ by an element in $S^{1}$ we may assume that $\left(w_{n-2}, u_{n-3, m+1}\right)$ and $\left(w_{n-2}, u_{n-3, m}\right)$ are real. Now we may write

$$
w_{n-2}=w_{n-2}^{\Lambda}+w_{n-2}^{\perp},
$$

where

$$
w_{n-2}^{\Lambda} \in \operatorname{ker}\left(\left(A_{n-3}-\Lambda \mathbb{I}\right)=\operatorname{span}\left\{\epsilon_{1}, \ldots, \epsilon_{m-1}\right\}\right.
$$

and

$$
w_{n-2}^{\perp} \in \operatorname{span}\left\{u_{n-3, m}, u_{n-3, m+1}\right\}=\operatorname{span}\left\{\epsilon_{m}, \epsilon_{m+1}\right\} .
$$

We have arranged for $w_{n-2}^{\perp}$ to be real. Choose $g \in \mathrm{U}(\mathrm{m}+1)$ with $g \epsilon_{m}=\epsilon_{m}$ and $g \epsilon_{m+1}=\epsilon_{m+1}$ such that

$$
g w_{n-2}^{\Lambda}=c^{\prime} \epsilon_{m-1}
$$

with $c^{\prime}=\left\|w_{n-2}^{\Lambda}\right\|$ as in the preceding step. Now change e to $g \mathbf{e}^{-1}$ and proceed to $w_{n-3}$.

We continue in this way until $\operatorname{ker}\left(A_{k}-\Lambda \mathbb{I}\right)=0$ and we enter the region $S_{2}$. The argument for this region is simpler and is left to the reader. Note that the vanishing of the angle variables says that all the coordinates $\left(w_{k}, u_{k-1, j}\right)$ in the eigenvector basis of $A_{k-1}$ have a common phase which can be eliminated by multiplication by an element of $S^{1}$; no conjugation is needed, so the preceding edges all remain real symmetric. However, the zero eigenvalue, which is unavoidable when we enter region $S_{1}$, causes new problems, and Lemma 7.2.15 is required.

Suppose then we have proved that $A_{m}$ is real (note that $A_{m}$ has rank $m$ ). We want to prove that $A_{m-1}$ is real. We know that

$$
A_{m}=A_{m-1}+r_{m+1} w_{m+1} \otimes w_{m+1}^{*},
$$

and since ker $A_{m}=\{0\}$, we have enough angle variables to prove that all coordinates of $w_{m+1}$ have a common phase. We clear this phase as before and move on to $A_{m-2}$. We have $A_{m-1}=A_{m-2}+r_{m} w_{m} \otimes$ $w_{m}^{*}$, and wish to prove that one can make $w_{m}$ real without destroying reality of $w_{n-1}, \ldots, w_{m+1}$. Write $w_{m}=w_{m}^{\perp}+w_{m}^{0}$ with $A_{m-1} w_{m}^{0}=0$ 
and $w_{m}^{\perp}$ orthogonal to ker $A_{m-2}$ (the latter has dimension 2). By the corollary to Lemma [7.2.15, we have $w_{m}^{0}=0$. Also, we have enough angle variables to conclude that the coordinates of $w_{m}^{\perp}$ relative to the eigenvectors of $A_{m-1}$ orthogonal to ker $A_{m-2}$ have a common phase. Thus, no conjugations are required to make $w_{m}$ real, and all preceding edges remain real symmetric. Now continue.

We remark that the proof could equally well be done by ascending induction; in that case, region $S_{1}$ would be the one requiring conjugations, while an overall scaling would do in $S_{2}, S_{3}$.

We are now ready to prove

\section{Proposition 7.2.18.}

$$
\left\{\theta_{i j}, \theta_{k l}\right\}=0
$$

Proof. Let $\mathbf{e} \in M_{\mathbf{r}}$ be given. By Corollary 7.2 .9 the bending deformations flows permute the level sets of the $\theta_{i j}$ 's transitively. Hence we may apply a bending $\phi$ to move e into $Z(\Theta)$. Since $\phi$ is symplectic and the Hamiltonian vector fields of the $\theta_{i j}$ are invariant under bending, we have

But by Proposition 7.2 .17

$$
\left\{\theta_{i j}, \theta_{k l}\right\}(\mathbf{e})=\left\{\theta_{i j}, \theta_{k l}\right\}(\phi \mathbf{e}) .
$$

$$
Z(\Theta)=M_{\mathbf{r}}\left(\mathcal{S}_{m+1}\right) .
$$

Hence by Lemma $\mathbf{7 . 2 . 1 2}$

$$
\left\{\theta_{i j}, \theta_{k l}\right\}=0
$$

\section{The DUAlity BetWEen THE BENDING SySTEMS AND THE GeL'FAnd-TSETLIN SYSTEMS ON GRASSMANNIANS}

In this section we use Gel'fand-MacPherson duality, following HK97 for the case of $m=1$, to show that the bending system is equivalent to the Gel'fand-Tsetlin integrable system (as defined in GS83) on a torus quotient of the Grassmannian $G\left(m+1, \mathbb{C}^{n}\right)$. This equivalence will explain the appearance and form of the Gel'fand-Tsetlin patterns in $\S 8$.

Our first goal is to construct a symplectomorphism $\Phi$ from $M_{\mathbf{r}}$ to a symplectic quotient of $G\left(m+1, \mathbb{C}^{n}\right)$ by the $n$-torus $T$ of diagonal matrices in $\mathrm{U}(\mathrm{n})$. This is the symplectic version of Gel'fand-MacPherson duality.

Let $\mathcal{M}$ denote the vector space of $(m+1) \times n$ complex matrices. We give $\mathcal{M}$ the Hermitean form (, ) defined by $(X, Y)=2 \operatorname{Tr}\left(X^{*} Y\right)$, and 
thus $\mathcal{M}$ is a symplectic vector space. The product group $\mathrm{U}(\mathrm{m}+1) \times$ $\mathrm{U}(\mathrm{n})$ acts isometrically and symplectically on $\mathcal{M}$. Denote the $i^{\text {th }}$ row (resp. $j^{\text {th }}$ column) of $N \in \mathcal{M}$ by $R_{i}$ (resp. $C_{j}$ ).

Proposition 8.1. The action of $U(n)$ has momentum map

$$
\mu_{U(n)}: \mathcal{M} \rightarrow \mathcal{H}_{n}, \quad \mu_{U(n)}: N \mapsto N^{*} N
$$

In particular, the momentum map for the T-action is

$$
\mu_{T}: N \mapsto\left(\left\|C_{1}\right\|^{2}, \ldots,\left\|C_{n}\right\|^{2}\right) .
$$

The momentum map for the $U(m+1)$ action is

$$
\mu_{U(m+1)}: \mathcal{M} \rightarrow \mathcal{H}_{m+1}, \quad \mu_{U(m+1)}: N \mapsto N N^{*} .
$$

Note that

$$
\mu_{\mathrm{U}(\mathrm{m}+1)}(N)=\sum_{i-1}^{n} C_{i} \otimes C_{i}^{*} .
$$

This will provide the connection with polygons.

We construct the desired symplectomorphism by computing the symplectic quotient corresponding to the $\mu_{T^{-}}$-level $\mathbf{r}$ and the $\mu_{\mathrm{U}(\mathrm{m}+1)}$ level $\Lambda \mathbb{I}$ in two different orders. If we first quotient with respect to $T$ with momentum level $\mathbf{r}$ and then with respect to $\mathrm{U}(\mathrm{m}+1)$ with momentum level $\Lambda$ II, we get the space $M_{\mathbf{r}}$. In order to see this, we note that the (left) action of $\prod_{1}^{n} \mathrm{U}(\mathrm{m}+1)$ on $\mathcal{M}$ (acting on the columns) commutes with the (right) action of $T$ (in fact one obtains a dual pair in the sense of Howe, see KKS78]). We first compute the symplectic quotient by $T$.

Lemma 8.2. (1) The momentum map $\mu_{(U(m+1))^{n}}$ induces an embedding of the symplectic quotient $\mu_{T}^{-1}(\mathbf{r}) / T$ into $\Pi_{1}^{n} \mathcal{H}_{m+1}$, with image $\prod_{1}^{n} \mathcal{O}_{r_{i}}$.

(2) The form on $\mu_{T}^{-1}(\mathbf{r}) / T$ induced by reducing the form $2 \operatorname{Im} X^{*} Y$ when carried over to $\prod_{1}^{n} \mathcal{O}_{r_{i}}$ agrees with the Kostant-Kirillov form $\omega_{K K}$.

Proof. The first statement follows because it is a general feature of dual pairs, see KKS78, that the momentum map for one action embeds the symplectic quotient of the other as an orbit in (the dual of) the Lie algebra of the first group. This principle, applied to the pair $(\mathrm{U}(\mathrm{m}+1))^{n} \times T$, implies the first statement in the lemma. The second follows from a straight-forward computation.

Thus we have identified the quotient by $T$ with the correct product of rank one orbits in $\mathcal{H}_{m+1}$. Clearly, after taking the symplectic quotient 
of this product by the diagonal action of $\mathrm{U}(\mathrm{m}+1)$ (at momentum level $\Lambda$ I), we obtain $M_{\mathrm{r}}$.

Suppose instead we first quotient with respect to $\mathrm{U}(\mathrm{m}+1)$ and momentum level $\Lambda \mathbb{I}$. We get the Grassmannian $G\left(m+1, \mathbb{C}^{n}\right)$ with a certain $\mathrm{U}(\mathrm{n})$-invariant symplectic structure.

Lemma 8.3. The momentum map $\mu_{U(n)}$ induces an embedding of the symplectic quotient $\mu_{U(m+1)}^{-1}(\Lambda \mathbb{I}) / U(m+1)$ into $\mathcal{H}_{n}$, with image the $U(n)$ orbit $\mathcal{O}_{\Lambda}$ consisting of those matrices that have eigenvalue $\Lambda$ with multiplicity $m+1$ and eigenvalue 0 with multiplicity $n-m-1$.

Proof. The argument is the analogous to the previous case, only this time we use the dual pair $\mathrm{U}(\mathrm{m}+1) \times \mathrm{U}(\mathrm{n})$.

Denote the torus quotient at momentum level $\mathbf{r}$ of the Grassmannian with the Kostant-Kirillov symplectic structure corresponding to $\Lambda$ by $\mathcal{M}_{\Lambda}$. We have now obtained the desired symplectomorphism $\Phi$ from $M_{\mathrm{r}}$ to $\mathcal{M}_{\Lambda}$.

Of course this symplectomorphism gives a Poisson isomorphism between the Poisson algebras of smooth functions of $M_{\mathrm{r}}$ and $\mathcal{M}_{\Lambda}$. However, we want to make this more explicit and to localize it. Let $\mathcal{M}_{\mathbf{r}, \Lambda}$ be the subset of $\mathcal{M}$ consisting of matrices $N$ such that $\left\|C_{j}\right\|^{2}=r_{j}$ and $N^{*} N=\Lambda \mathbb{I}$. Thus we have $\mathrm{U}(\mathrm{m}+1) \times T$ quotient mappings $\pi_{1}: \mathcal{M}_{\mathrm{r}, \Lambda} \rightarrow$ $M_{\mathbf{r}}$ (first quotient by $T$ then by $\mathrm{U}(\mathrm{m}+1)$ ) and $\pi_{2}: \mathcal{M}_{\mathbf{r}, \Lambda} \rightarrow \mathcal{M}_{\Lambda}$ (first quotient by $\mathrm{U}(\mathrm{m}+1)$ then by $T)$. We use the mappings $\pi_{1}$ and $\pi_{2}$ to realize (and localize) the Poisson isomorphism $\Phi$ from above. Let $f$ be a function which is smooth on an open subset of $M_{\mathbf{r}}$. Use $\pi_{1}$ to pull $f$ back to a $\mathrm{U}(\mathrm{m}+1) \times T$-saturated open subset of $\mathcal{M}_{\mathbf{r}, \Lambda}$. Since $\pi_{2}$ is a quotient map and $\pi_{1}^{*} f$ is invariant under $\mathrm{U}(\mathrm{m}+1)$, we can first descend it to to a $T$-saturated open subset of the Grassmannian, then to the torus quotient of that open set, which is an open subset of $\mathcal{M}_{\Lambda}$. We note that $\Phi$ is determined by the equation

$$
\Phi\left(\pi_{1}(N)\right)=\pi_{2}(N) .
$$

We now briefly review the Gel'fand-Tsetlin integrable system - for the details see GS83. As before, we identify the space $\mathcal{H}_{n}$ of $n \times n$ Hermitean matrices with the dual of the Lie algebra of $U(n)$. We now construct $n(n+1) / 2$ Poisson commuting functions on $\mathcal{H}_{n}$ which are smooth on a dense open subset. Let $X \in \mathcal{H}_{n}$. Let $\beta_{i}(X)$ be the principal $i \times i$ diagonal block. Define $\gamma_{i j}$ on $\mathcal{H}_{n}$ by

$$
\gamma_{i j}(X)=\lambda_{j}\left(\beta_{i}(X)\right)
$$

where $\lambda_{j}$ is the $j^{\text {th }}$ eigenvalue of the block. As usual, we assume that the eigenvalues of the $i^{\text {th }}$ block are arranged in nonincreasing order. It 
is proved in GS83 that the $\gamma_{i j}$ 's Poisson commute. We note that the $\gamma_{n j}$ are Casimirs. The restrictions of the remaining Gel'fand-Tsetlin Hamiltonians to generic orbits are functionally independent and give rise to integrable system on such orbits. The eigenvalues of the blocks interlace and can be arranged in a "Gel'fand-Tsetlin" pattern, shown here for $n=6$.

$\begin{array}{ccccccccccc}\gamma_{61} & & \gamma_{62} & & \gamma_{63} & & \gamma_{64} & & \gamma_{65} & & \gamma_{66} \\ & \gamma_{51} & & \gamma_{52} & & \gamma_{53} & & \gamma_{54} & & \gamma_{55} & \\ & & \gamma_{41} & & \gamma_{42} & & \gamma_{43} & & \gamma_{44} & & \\ & & \gamma_{31} & & \gamma_{32} & & \gamma_{33} & & & \\ & & & \gamma_{21} & & \gamma_{22} & & & \\ & & & & & \gamma_{11} & & & & \end{array}$

\section{Figure 3}

Since we are dealing with a degenerate orbit here (the Grassmannian), many of the $\gamma_{i j}$ 's (at the ends of the rows) will be zero (see Remark 8.5 below, and Figure 2 above). The next proposition, combined with the earlier sections, shows how to extract a functionally independent set of Gel'fand-Tsetlin Hamiltonians and obtain angle variables for the Gel'fand-Tsetlin system on the Grassmannian.

Proposition 8.4. $\Phi^{*} \gamma_{i j}=\lambda_{i j}$.

Proof. Let $\mathbb{I}_{k}$ be the diagonal $n$ by $n$ matrix whose first $k$ eigenvalues are equal to 1 and last $n-k$ eigenvalues are equal to 0 . We use $\mathbb{I}_{k}$ to "truncate" $N, N^{*} N$ and $N N^{*}$. Put $N_{k}:=N \mathbb{I}_{k}$. Then

$$
\begin{aligned}
\mu_{\mathrm{U}(\mathrm{n})}\left(N_{k}\right) & =\mathbb{I}_{k} N^{*} N \mathbb{I}_{k} \\
\mu_{\mathrm{U}(\mathrm{m}+1)}\left(M_{k}\right) & =N \mathbb{I}_{k} \mathbb{I}_{k} N^{*} .
\end{aligned}
$$

The matrix on the first line is $\beta_{k}\left(N^{*} N\right)$, the principal $k$ by $k$ block of the $n \times n$ matrix $N^{*} N$, and the matrix on the second line is the diagonal $A_{k-1}=C_{1} C_{1}^{*}+C_{2} C_{2}^{*}+\cdots+C_{k} C_{k}^{*}$. The matrices $\mathbb{I}_{k} N^{*} N \mathbb{I}_{k}$ and $N \mathbb{I}_{k} \mathbb{I}_{k} N^{*}$ have the same nonzero eigenvalues. But the eigenvalues of the second matrix are the bending Hamiltonians $\lambda_{k j}$, and the eigenvalues of the first matrix are the Gel'fand-Tsetlin Hamiltonians $\gamma_{k j}$. Finally we observe that

$$
\begin{aligned}
\gamma_{i j}\left(\Phi\left(\pi_{1}(M)\right)\right) & =\gamma_{i j}\left(\pi_{2}(M)\right)=\lambda_{j}\left(\beta_{i}\left(\pi_{M}\right)\right) \\
& =\lambda_{j}\left(A_{i}\left(\pi_{1}(M)\right)\right)=\lambda_{i j}\left(\pi_{1}(M)\right) .
\end{aligned}
$$

We conclude this section with three remarks. 
Remark 8.5. Proposition 8.4 explains the appearance of Gel'fand-Tsetlin patterns in connection with the bending Hamiltonians. The appearance of the zeroes at the end of the rows in our patterns is explained because the Gel'fand-Tsetlin system in question is defined on a subset of the Hermitean matrices of rank at most $m+1$. Hence $\gamma_{i j}=0, j>m+1$.

Remark 8.6. The reconstruction process in $\S 9$ may be interpreted as saying that the class of patterns introduced in $\S 8$ is precisely the class corresponding to Hermitean matrices of the form $N^{*} N$, where $N$ is as above.

Remark 8.7. Fixing the row sums in the patterns in $\S 8$ to be partial sums of the $r_{j}$ corresponds to taking the quotient of the Grassmannian by $T$ (at level $\mathbf{r}$ ).

\section{Pieri's Formula AND The DUAlity AT ThE QUANTUM LEVEL}

In this section we will assume that the $r_{i}$ 's are (positive) integers and that $\Lambda=\left(r_{1}+\cdots+r_{n}\right) /(m+1)$ is an integer. The orbit $\mathcal{O}_{r_{i}}$ then corresponds under geometric quantization to the irreducible representation $\mathcal{S}^{r_{i}}(V)$ of $\mathrm{U}(\mathrm{m}+1)$, where $V$ denotes the standard (or vector) representation of $\mathrm{U}(\mathrm{m}+1)$ on $\mathbb{C}^{m+1}$ and $\mathcal{S}^{r_{i}}(V)$ the $r_{i}^{\text {th }}$ symmetric power.

The (classical) duality result of the last section should have a quantum version. We note that the duality connected an integrable system (bending) on a symplectic quotient of $\prod_{1}^{n} \mathcal{O}_{r_{i}}$ by the diagonal action of $\mathrm{U}(\mathrm{m}+1)$ and an integrable system (Gel'fand-Tsetlin) on a torus quotient of the Grassmannian $G(m+1, n)$. Thus, according to geometric quantization, at the quantum level we would expect a relation between an $n$-fold tensor product multiplicity for $\mathrm{GL}(\mathrm{m}+1, \mathbb{C})$ and a weight multiplicity for a Cartan power of the the $(m+1)^{\text {st }}$ exterior power of $\mathrm{GL}(\mathrm{n}, \mathbb{C})$. The bending system provides a (singular) real polarization of the space $M_{\mathbf{r}}$, the symplectic quotient (at level $\Lambda$ II) of $\prod_{i} \mathcal{O}_{r_{1}}$. Thus the number of lattice points in the momentum polyhedron $\mathbf{P}$ for bending should be equal to the multiplicity of the the 1-dimensional representation $(d e t)^{\Lambda}$ in $\otimes_{1}^{n} \mathcal{S}^{r_{i}}(V)$. But on the other hand, the Gel'fand-Tsetlin system is a real polarization of the torus quotient of the Grassmannian (at level $\mathbf{r}$ ) where the Grassmannian is given the symplectic structure which corresponds to the orbit of $\mathrm{U}(\mathrm{n})$ through the diagonal matrix with $m+1 \Lambda$ 's and $n-m-1$ zeroes. Thus the above number of lattice points should also be the multiplicity of the $\mathbf{r}^{\text {th }}$ weight space in $C^{\Lambda} \bigwedge^{m+1} V$, the $\Lambda^{\text {th }}$ Cartan power of the $m+1$-st exterior power of the vector representation $V$ of $\mathrm{GL}(\mathrm{n}, \mathbb{C})$. (We recall that if $W^{\nu}$ is a representation with highest weight $\nu$, then the $p$-th Cartan power $C^{p} W^{\nu}$ is 
the irreducible representation with highest weight $p \nu$ ). This equality of multiplicities predicted is in fact true, and will be proved below.

Remark 9.1. It is unfortunate that the theory of geometric quantization using a real polarization is not sufficiently well developed to allow one to deduce theorems in representation theory from equalities of numbers of lattice points in momentum polyhedra. At this time we can only regard such equalities as predictions of theorems in representation theory.

We first note how the interlacing of the spectra of the perturbed matrix and the unperturbed matrix (see $\S 8$ )from the Weinstein-Aronszajn formula predicts Pieri's formula in representation theory.

9.1. The Weinstein-Aronszajn and Pieri formulas. We recall Pieri's formula for tensoring an irreducible polynomial representation of $\mathrm{U}(\mathrm{m}+1)$ with a symmetric power of the vector representation [FH, §A.1].

Theorem 9.1.1 (Pieri's Formula). Let $\lambda=\left(\lambda_{1}, \ldots, \lambda_{m+1}\right)$ be the highest weight of the polynomial representation $V\left(\lambda_{1}, \ldots, \lambda_{m+1}\right)$ of $U(m+1)$. Let $k$ be a positive integer. Then

$$
V\left(\lambda_{1}, \ldots, \lambda_{m+1}\right) \otimes \mathcal{S}^{k}(V)=\oplus V\left(\nu_{1}, \ldots, \nu_{m+1}\right)
$$

where the sum is taken over all dominant $\nu=\left(\nu_{1}, \ldots, \nu_{n}\right)$ satisfying

$$
\nu_{1} \geq \lambda_{1} \geq \nu_{2} \geq \ldots \geq \nu_{m+1} \geq \lambda_{m+1} \geq 0
$$

and

$$
\sum_{i=1}^{m+1} \nu_{i}=\sum_{i=1}^{m+1} \nu_{i}+k .
$$

This is of course Proposition 6.1.3 restricted to integer eigenvalues. If $A \in \mathcal{O}_{\lambda}$, then the spectrum of the rank one perturbation $A+k w \otimes w^{*}$ satisfies the interlacing and row sum conditions of the Pieri formula.

9.2. Duality at the quantum level. In this subsection we prove the theorem from representation theory that is predicted by the equality (of the numbers of lattice points) of the momentum polyhedra for bending and Gel'fand-Tsetlin. The required facts from representation theory can be found in $[\mathrm{FH}]$ and $[\mathrm{Ze}]$.

Theorem 9.2.1. The multiplicity of the 1-dimensional representation $(\text { det })^{\Lambda}$ in $\otimes_{1}^{n} \mathcal{S}^{r_{j}}(V)$ is equal to the multiplicity of the weight $\mathbf{r}$ in the irreducible representation $C^{\Lambda} \bigwedge^{m+1} V$ of $U(n)$. This common multiplicity is in fact equal to the number of lattice points in $\mathbf{P}$.

The theorem will be a consequence of the next three lemmas. We will need 
Definition 9.2.2. Let $\lambda$ be an l-tuple of positive integers and $\mu$ be a partition. Then the Kostka number $K_{\mu \lambda}$ is the number of ways to fill in the Young diagram corresponding to $\mu$ with $\lambda_{1} 1$ 's, $\lambda_{2} 2$ 's, ..., $\lambda_{l}$ $l$ 's so that the rows are weakly increasing and the columns are strongly increasing.

By applying Pieri's formula iteratively one gets [FH], (A.9)]:

\section{Lemma 9.2.3.}

$$
\mathcal{S}^{r_{1}}(V) \otimes \mathcal{S}^{r_{2}}(V) \otimes \cdots \otimes \mathcal{S}^{r_{n}}(V)=\oplus_{\mu} K_{\mu \mathrm{r}} V(\mu)
$$

We obtain

Corollary 9.2.4. The multiplicity of the 1-dimensional representation $(\text { det })^{\Lambda}$ in $\otimes_{1}^{n} \mathcal{S}^{r_{j}}(V)$ is equal to the Kostka number $K_{\Lambda\left(1^{m+1}\right) \mathbf{r}}$.

Here the symbol $\Lambda\left(1^{m+1}\right)$ means the partition $(\Lambda, \Lambda, \ldots, \Lambda)$ (there are $m+1 \Lambda$ 's). The corresponding Young diagram has $m+1$ rows and $\Lambda$ columns.

In order to compare $K_{\Lambda\left(1^{m+1}\right) \mathbf{r}}$ with the multiplicity of the weight $\mathbf{r}$ in the irreducible representation $C^{\Lambda} \bigwedge^{m+1} V$ of $\mathrm{U}(\mathrm{n})$ we recall there is a basis for an irreducible representation of $G L(n)$ labeled by semistandard Young tableaux. Suppose the highest weight of the representation is $\mu$. We also use $\mu$ to denote the Young diagram associated to $\mu$. A semistandard filling of $\mu$ is an assignment of the integers between 1 and $n$ to the boxes of $\mu$ such that the rows are weakly increasing and the columns are strongly increasing. The associated basis is a weight basis, and the weight of of the basis vector corresponding to a semistandard tableau is $\left(k_{1}, \ldots, k_{n}\right)$, where $k_{i}$ is the number of $i$ 's in the tableau. Thus we have proved

Lemma 9.2.5. $K_{\Lambda\left(1^{m+1}\right) \mathbf{r}}$ is also the multiplicity of the weight $\mathbf{r}$ in $C^{\Lambda} \wedge^{m+1} V$ of $G L(n)$.

It still remains to prove that the number of lattice points in $\mathbf{P}$ is the common multiplicity.

To see this we recall that there is an orthonormal basis (the Gel'fandTsetlin basis) for the irreducible representation $C^{\Lambda} \wedge^{m+1} V$ indexed by Gel'fand-Tsetlin patterns whose top row consists of $m+1 \Lambda$ 's and $n-m-1$ zeroes. Moreover, this basis is a weight basis, and the weight of a basis vector corresponding to a Gel'fand-Tsetlin pattern is given by the differences in the row sums starting with the bottom entry in the pattern. Thus we have

Lemma 9.2.6. The number of lattice points in $\mathbf{P}$ is equal to the dimension of the $\mathbf{r}^{\text {th }}$ weight space in $C^{\Lambda} \bigwedge^{m+1} V$. 
It follows that the count of lattice points in $\mathbf{P}$ gives the correct answer for both multiplicities.

Remark 9.2.7. One might ask whether there is a direct combinatorial argument to establish the last lemma above, i.e. that the number of semistandard Young tableaux of weight $\mathbf{r}$ is equal to the number of Gel'fand-Tsetlin patterns of weight $\mathbf{r}$. In fact, there is a one to one weight preserving correspondence between semistandard Young tableaux and Gel'fand-Tsetlin patterns, see GZ86].

\section{Appendix: Bending and Hitchin Hamiltonians}

In $[\mathrm{AHH}$, the authors constructed a duality between integrable systems on certain pairs of finite dimensional coadjoint orbits of loop groups. The spaces considered in Chapter 8 above belong to their family. However we shall show below that the Hamiltonians considered in $\mathrm{AHH}$ are different from ours even for the case of polygons in $\mathbb{R}^{3}$ i.e. $m=1$. The point is that our Hamiltonians depend on a triangulation of the polygon by diagonals (equivalently, the GelfandTsetlin decomposition into increasing principal diagonal blocks). This introduces an asymmetry which is not present in the theory of $[\mathrm{AHH}$. We will not review the theory of $\mathrm{AHH}$ here but will instead review how one obtains integrable systems on $M_{\mathbf{r}}$ by associating a matrix $A(z)$ with entries which are polynomials in a complex variable $z$ to a point e $\in \widetilde{M}_{\mathbf{r}}$. The construction of $\mathrm{AHH}$ is more general but reduces to the above for the case in hand.

We will refer to the resulting Hamiltonians as Hitchin Hamiltonians although the construction we are about to describe antedated and is a very special case of Hitchin's construction of integrable systems associated to Higgs fields. We will follow the notation of [Hi], pages $46-52$.

10.1. Hitchin Hamiltonians. We will describe the construction of Hitchin Hamiltonians for the case considered in this paper. So let e $\in \widetilde{M}_{\mathbf{r}}$. Choose $n$ points $\alpha_{1}, \alpha_{2}, \cdots, \alpha_{n}$. Put $p(z)=\prod_{i=1}^{n}\left(z-\alpha_{i}\right)$ and define a matrix-valued polynomial $A(z)$ by

$$
A(z)=p(z) \sum_{i=1}^{n} \frac{e_{i}}{z-\alpha_{i}} .
$$

We then consider the characteristic polynomial $\operatorname{det}(w \mathbb{I}-A(z))$ of $A(z)$. The coefficients of the characteristic polynomial (considered as functions on $M_{\mathbf{r}}$ ) are the Hitchin Hamiltonians. In the case in which $m=1$ 
it suffices to consider the functions

$$
H_{j}(\mathbf{e})=\sum_{i \neq j} \frac{e_{i} \cdot e_{j}}{\alpha_{i}-\alpha_{j}}, 1 \leq j \leq n .
$$

We will now examine when the bending flows leave invariant the Hitchin Hamiltonians for the special case $m=1$. We note that if $f$ is a smooth function of $M_{\mathbf{r}}$ in order to compute its derivative along a bending flow we may choose any lift of $f$ to $\widetilde{M}_{\mathbf{r}}$ and any lift of the bending flow to $\widetilde{M_{\text {r }}}$.

10.2. Bending Hamiltonians are not Hitchin Hamiltonians. In this section we will prove

Proposition 10.2.1. If $\alpha_{1}, \cdots, \alpha_{n}$ are distinct and $n \geq 5$, then the diagonal $d_{13}:=\left\|e_{1}+e_{2}\right\|$ does not belong to the Hitchin system.

The proposition will follow from the next two lemmas. Let $\phi_{t}$ be the bending flow associated to diagonal $d_{13}$. We will lift $d_{13}$ to $\widetilde{M}_{\mathbf{r}}$ and lift $\phi_{t}$ to $\widetilde{M}_{\mathbf{r}}$ so that the edges $e_{1}$ and $e_{2}$ are rotated around the diagonal $e_{1}+e_{2}$ and the remaining edges are left fixed. We will continue to use $\phi_{t}$ for the lifted flow.

Lemma 10.2.2. Let $i>2$ and $c$ be a constant. Then the function $g(\mathbf{e}):=c e_{1} \cdot e_{i}$ is invariant under the flow $\phi_{t} \Leftrightarrow c=0$.

Proof. We have

$$
\dot{e_{1}}=e_{2} \times e_{1}, \dot{e_{2}}=e_{1} \times e_{2} \text { and } \dot{e_{i}}=0, i>2 .
$$

Hence, if $i>2$ we have

$$
\frac{d}{d t} c e_{1} \cdot e_{i}=c\left(e_{2} \times e_{1}\right) \cdot e_{i} \equiv 0 .
$$

But there exist polygons such that the scalar triple $\left(e_{2} \times e_{1}\right) \cdot e_{i}$ is nonzero. Consequently $c=0$.

In the proof of the next two lemmas we will use the following notation. If $f$ and $g$ are two smooth functions on $\widetilde{M}_{\mathbf{r}}$ we will write $f \equiv g$ if $f$ and $g$ differ by a function that is invariant under $\phi_{t}$.

Recall $H_{n}(e)$ is the Hitchin Hamiltonian defined by

$$
H_{n}(e)=\sum_{i=1}^{n-1} \frac{e_{i} \cdot e_{n}}{\alpha_{i}-\alpha_{n}} .
$$

Lemma 10.2.3. $H_{n}$ is invariant under $\phi_{t} \Leftrightarrow \alpha_{1}=\alpha_{2}$. 
Proof. We have, since $e_{i} \cdot e_{n}$ is invariant under $\phi_{t}$ for $i>2$ (since $e_{i}$ is)

$$
\begin{aligned}
H_{n}(e) & \equiv \frac{e_{1} \cdot e_{n}}{\alpha_{1}-\alpha_{n}}+\frac{e_{2} \cdot e_{n}}{\alpha_{2}-\alpha_{n}} \\
& =\frac{e_{1} \cdot e_{n}}{\alpha_{1}-\alpha_{n}}+\frac{\left(\left(e_{1}+e_{2}\right)-e_{1}\right) \cdot e_{n}}{\alpha_{2}-\alpha_{n}} \\
& \equiv\left(\frac{1}{\alpha_{1}-\alpha_{n}}-\frac{1}{\alpha_{2}-\alpha_{n}}\right) e_{1} \cdot e_{n} .
\end{aligned}
$$

The last line follows because $e_{1}+e_{2}$ is invariant under $\phi_{t}$.

By the previous lemma we find that $H_{n}(e)$ is invariant if and only if

$$
\frac{1}{\alpha_{1}-\alpha_{n}}-\frac{1}{\alpha_{2}-\alpha_{n}}=0
$$

There remains the possibility that all the bending Hamiltonians could be Hitchin Hamiltonians for a singular curve. We will now show that this cannot happen (for the case $n=5$ ). Suppose then $n=5$. Let $\phi_{t}$ be the bending flow along the diagonal $e_{1}+e_{2}$ and $\psi_{t}$ be the bending flow along the diagonal $e_{1}+e_{2}+e_{3}$. We will lift $\phi_{t}\left(\right.$ resp. $\left.\psi_{t}\right)$ to $\widetilde{M}_{\mathbf{r}}$ so that only the first two (resp. three) edges are moved. We will now use the notation $f \equiv g$ to denote that $f$ and $g$ differ by a function that is invariant under both flows.

Lemma 10.2.4. Suppose the Hitchin Hamiltonian $\mathrm{H}_{5}$ is invariant under both bending flows $\phi_{t}$ and $\psi_{t}$. Then the first three $\alpha_{i}$ 's are equal.

Proof. Since $e_{4}$ and $e_{5}$ are invariant under both flows we have

$$
\begin{aligned}
H_{5}(e) & \equiv \frac{e_{1} \cdot e_{5}}{\alpha_{1}-\alpha_{5}}+\frac{e_{2} \cdot e_{5}}{\alpha_{2}-\alpha_{5}}+\frac{e_{3} \cdot e_{5}}{\alpha_{3}-\alpha_{5}} \\
& =\frac{e_{1} \cdot e_{5}}{\alpha_{1}-\alpha_{5}}+\frac{e_{2} \cdot e_{5}}{\alpha_{2}-\alpha_{5}}+\frac{\left(\left(e_{1}+e_{2}+e_{3}\right)-\left(e_{1}+e_{2}\right)\right) \cdot e_{5}}{\alpha_{3}-\alpha_{5}} \\
& \equiv\left(\frac{1}{\alpha_{1}-\alpha_{5}}-\frac{1}{\alpha_{3}-\alpha_{5}}\right) e_{1} \cdot e_{5}+\left(\frac{1}{\alpha_{2}-\alpha_{5}}-\frac{1}{\alpha_{3}-\alpha_{5}}\right) e_{2} \cdot e_{5} .
\end{aligned}
$$

But by Lemma 10.2.3 we have

$$
\frac{1}{\alpha_{1}-\alpha_{5}}=\frac{1}{\alpha_{2}-\alpha_{5}} \text {. }
$$

Consequently we have

$$
H_{5}(e) \equiv\left(\frac{1}{\alpha_{1}-\alpha_{5}}-\frac{1}{\alpha_{3}-\alpha_{5}}\right)\left(e_{1}+e_{2}\right) \cdot e_{5} .
$$


We put $c$ equal to the coefficient of $\left(e_{1}+e_{2}\right) \cdot e_{5}$ act by $\psi_{t}$ and differentiate to obtain

$$
\frac{d}{d t} c\left(e_{1}+e_{2}\right) \cdot e_{5}=c\left(\left(e_{1}+e_{2}+e_{3}\right) \times\left(e_{1}+e_{2}\right)\right) \cdot e_{5}=0 .
$$

But there exist polygons such that the number $\left(\left(e_{1}+e_{2}+e_{3}\right) \times\left(e_{1}+\right.\right.$ $\left.\left.e_{2}\right)\right) \cdot e_{5}$ is nonzero. Consequently $c=0$.

Hence $\alpha_{1}=\alpha_{3}$ and the first three $\alpha_{i}$ 's are equal.

Now we prove that if $H_{1}(e)$ is also invariant under $\phi_{t}$ and $\psi_{t}$ then all the $\alpha_{i}$ 's are equal.

Lemma 10.2.5. Suppose the Hitchin Hamiltonian $H_{1}$ is invariant under both bending flows $\phi_{t}$ and $\psi_{t}$. Then the last three $\alpha_{i}$ 's are equal.

Proof. Lift $\psi_{t}$ from $M_{\mathbf{r}}$ to $\widetilde{M}_{\mathbf{r}}$ so that it rotates the triangle with edges $e_{1}+e_{2}+e_{3}, e_{4}$ and $e_{5}$ around the diagonal $e_{1}+e_{2}+e_{3}$ (so $\psi_{t}$ leaves $e_{1}, e_{2}$ and $e_{3}$ fixed). Repeat the argument in Lemma 10.2.3 to find $\alpha_{4}=\alpha_{5}$. Now lift $\phi_{t}$ to $\widetilde{M}_{\mathrm{r}}$ so that it rotates the quadrilateral with edges $e_{5}, e_{4}, e_{3}$ and $e_{1}+e_{2}$ around the diagonal $e_{1}+e_{2}$ (so $\phi_{t}$ leaves $e_{1}$ and $e_{2}$ fixed). Repeat the argument of Lemma 10.2.4 to find the last three $\alpha_{i}$ 's are equal.

We can now show for pentagons the bending systems and the Hitchin systems never coincide.

Theorem 10.2.6. For the case $n=5$, the Hitchin system never coincides with the bending system.

Proof. If the systems coincide then $H_{1}$ and $H_{5}$ are invariant under both bending flows and all the $\alpha_{i}$ 's are equal. But in this case we obtain

$$
A(z)=\sum_{i=1}^{5} \frac{e_{i}}{z-\alpha_{i}}=\frac{1}{z-\alpha_{1}} \sum_{i=1}^{5} e_{i}=0 .
$$

Hence $\operatorname{det}(w \mathbb{I}-A(z))=w^{2}$ and there are no nontrivial Hitchin Hamiltonians.

\section{REFERENCES}

[AHH] M. R. Adams, J. Harnad and J. Hurtubise, Dual moment maps into loop algebras, Letters in Mathematical Physics, 20(1990), 299-308.

[BeSch] S. Berceanu and M. Schlichenmaier, Coherent states, embeddings, polar divisors and Cauchy formulas, Journal of Geometry and Physics, 34(2000), 336-368.

[BrJa] Th. Bröcker and K. Jänich, Introduction to Differential Topology, Cambridge University Press, 1982. 
[DM86] P. Deligne and G.D. Mostow, Monodromy of hypergeometric functions and nonlattice integral monodromy, Publ. Math. IHES, 63 (1986), 5-90.

[DO] I. Dolgachev and D. Ortland, Point Sets in Projective Space and Theta Functions, Astérisque, 165 (1988).

[FH] W. Fulton and J. Harris, Representation Theory, A First Course, Graduate Texts in Mathematics, no. 129, Springer-Verlag New YorkBerlinHeidelberg, 1991.

[GGMS] I. M. Gel'fand, R. M. Goresky, R. D. MacPherson and V. V. Serganova, Combinatorial geometries, convex polyhedra and Schubert cells, Advances in Math., 63 (1987), 301-316.

[Go] W. M. Goldman, Complex Hyperbolic Geometry, Oxford Mathematical Monographs, Clarendon Press, Oxford.

[GZ86] I. Gel'fand and A. Zelevinsky, Multiplicities and proper bases for $g l_{n}$, in Group Theoretical Methods in Physics, Proceedings of the Third Yurmala Seminar, M. A. Markov, V. I. Mank'o, V. V. Dodonov (editors) VNU Science Press, Utrecht, The Netherlands (1986), 147-159.

[Goldin] R. F. Goldin, The cohomology ring of weight varieties and polygon spaces, Adv.Math., 160 (2001), 175-204.

[GS83] V. Guillemin and S. Sternberg, The Gel'fand-Cetlin system and quantization of the complex flag manifolds, J. Functional Anal., 52 (1983), 106-128.

[HM] Th. Hangan and G. Masala, A geometric interpretation of the shape invariant for geodesic triangles in complex projective spaces, Geom. Dedicata, 49 (1994), 129-134.

[HK97] J.- C. Hausmann and A. Knutson, Polygons spaces and Grassmannians, Enseign. Math., 43 (1997), 173-198.

[HL94] P. Heinzner and F. Loose, Reduction of complex Hamiltonian $G$-spaces, GAFA, 4, no. 3 (1994), 288-297.

[Hi] N. J. Hitchin, Riemann surfaces and integrable systems, in Integrable Systems, Twistors, Loop Groups and Riemann Surfaces, Oxford Graduate Texts in Mathematics, Clarendon Press, Oxford, 1999.

$[\mathrm{Hu}] \mathrm{Yi} \mathrm{Hu}$, The geometry and topology of quotient varieties of torus actions, Duke Math. J., 68, no. 1 (1992), 151-184.

[KM95] M. Kapovich and J. Millson, On the moduli space of polygons in the Euclidean plane, J. Differential Geom., 42 (1995), 430-464.

[KM96] M. Kapovich and J. Millson, The symplectic geometry of polygons in Euclidean space, J. Diff. Geom., 44 (1996), 479-513.

[KM01] M. Kapovich and J. Millson, Quantization of bending deformations of polygons in $\mathbb{E}^{3}$, hypergeometric integrals and the Gassner representation, Canad. Math. Bull., 44, (2001), 36-60.

[Kato] T. Kato, Perturbation Theory for Linear Operators, Die Grundlehren der mathematischen Wissenschaften 132, Springer Verlag.

[KKS78] D. Kazhdan, B. Kostant and S. Sternberg, Hamiltonian group actions and dynamical systems of Calogero type, Comm. Pure Appl. Math., 31 (1978), 481-508.

[KN79] G. Kempf and L. Ness, The length of vectors in representation spaces, in: Algebraic Geometry, Proceedings, Copenhagen 1978, Springer Lecture Notes in Mathematics 732 (1979), 233-243. 
[Ki] F. C. Kirwan, Cohomology of Quotients in Symplectic and Algebraic Geometry, Princeton Mathematical Notes 31, Princeton University Press, 1984.

[Kly92] A. Klyachko, Spatial polygons and stable configurations of points on the projective line, in: Algebraic Geometry and its Applications, Proceedings of the 8th Algebraic Geometry Conference, Yaroslavl' 1992, A. Tikhomirov and A. Tyurin (Eds.), Vieweg, 67-84.

[Kly98] A. Klyachko, Stable bundles, representation theory and Hermitean operators, Selecta Mathematica, 4 (1998), 419-445.

[MR] J. E. Marsden and T. S. Ratiu, Introduction to Mechanics and Symmetry, Springer-Verlag, New York-Berlin-Heidelberg, 1994.

[Ne84] L. Ness, A stratification of the null cone via the moment map, Amer. J. Math., 106 (1984), 1281-1329.

[MFK] D. Mumford, J. Fogarty, F. Kirwan, Geometric Invariant Theory, third enlarged edition, Ergebnisse der Mathematik und ihre Grenzgebiete 34, Springer Verlag, 1991.

[Sj95] R. Sjamaar Holomorphic slices, symplectic reduction and multiplicities of representations, Annals of Math., 141, (1995), 87-129.

[Ze] D. P. Želobenko, Compact Lie Groups and Their Representations, AMS, 1973.

Department of Mathematics, The University of Arizona, Tucson, AZ 85721

E-mail address: flaschka@math.arizona.edu

Department of Mathematics, University of Maryland, College Park, MD 20742

E-mail address: jjm@math.umd.edu 